\title{
Improved resolution backscatter measurements with the SeaWinds pencil-beam scatterometer
}

\author{
David G. Long \\ david_long@byu.edu \\ Michael W. Spencer \\ Chialin T. Wu
}

Follow this and additional works at: https://scholarsarchive.byu.edu/facpub

Part of the Electrical and Computer Engineering Commons

\section{Original Publication Citation}

Spencer, M. W., C. Wu, and D. G. Long. "Improved Resolution Backscatter Measurements with the SeaWinds Pencil-Beam Scatterometer." Geoscience and Remote Sensing, IEEE Transactions on 38.1 (2): 89-14

\section{BYU ScholarsArchive Citation}

Long, David G.; Spencer, Michael W.; and Wu, Chialin T., "Improved resolution backscatter measurements with the SeaWinds pencil-beam scatterometer" (2000). Faculty Publications. 606.

https://scholarsarchive.byu.edu/facpub/606

This Peer-Reviewed Article is brought to you for free and open access by BYU ScholarsArchive. It has been accepted for inclusion in Faculty Publications by an authorized administrator of BYU ScholarsArchive. For more information, please contact ellen_amatangelo@byu.edu. 


\title{
Improved Resolution Backscatter Measurements with the SeaWinds Pencil-Beam Scatterometer
}

\author{
Michael W. Spencer, Chialin Wu, and David G. Long, Senior Member, IEEE
}

\begin{abstract}
The SeaWinds scatterometer was launched on the NASA QuikSCAT spacecraft in June 1999 and is planned for the Japanese ADEOS-II mission in 2000. In addition to generating a global Ku-band backscatter data set useful for a variety of climate studies, these flights will provide ocean-surface wind estimates for use in operational weather forecasting. SeaWinds employs a compact "pencil-beam" design rather than the "fan-beam" approach previously used with SASS on Seasat, NSCAT on ADEOS-I, and the AMI scatterometer on ERS-1, 2. As originally envisioned and reported, the resolution of the SeaWinds backscatter measurements were to be antenna-beamwidth limited. In order to satisfy an emerging demand for higher resolution backscatter data, however, the SeaWinds signal-processing design has been significantly modified. Here, the various options considered for improving the resolution of the SeaWinds measurements are discussed, and the selected hardware modification (the addition of deramp processing for range discrimination) is described. The radar equation specific to a rotating pencil-beam scatterometer with digital range filtering is developed, and the new challenges associated with calibrating the resulting improved resolution measurements are discussed. A formulation for assessing the variance of the measurements due to fading and thermal noise is presented. Finally, the utility of improved resolution SeaWinds measurements for land and ice studies is demonstrated by simulated enhanced-resolution imaging of a synthetic Earth backscatter scene.
\end{abstract}

Index Terms - Radar, scatterometry, sea winds.

\section{INTRODUCTION AND BACKGROUND}

$\mathbf{S}$ PACEBORNE wind scatterometry has become an increasingly important tool in the effort to monitor the Earth's climate, forecast weather, and study ocean-atmosphere interaction. To continue and expand upon the foundation provided by the Seasat-A scatterometer (SASS) and the NASA scatterometer (NSCAT), NASA has developed the SeaWinds instrument, which is scheduled for two flights. The first is on the dedicated QuikSCAT mission in June 1999, and the second is aboard the second Japanese Advanced Earth Observation Satellite (ADEOS-II) in 2000.

\section{A. SeaWinds Scatterometer Design}

As with all scatterometers, SeaWinds will obtain an estimate of the wind vector by measuring the ocean-surface radar

Manuscript received September 28, 1998; revised February 26, 1999. This work was supported by the Jet Propulsion Laboratory, California Institute of Technology, Pasadena, CA, under a contract with the National Aeronautics and Space Administration.

M. W. Spencer and C. Wu are with the Jet Propulsion Laboratory, California Institute of Technology, Pasadena, CA 91109-8099 USA (e-mail: mws@shiokaze.jpl.nasa.gov).

D. G. Long is with the Department of Electrical and Computer Engineering, Brigham Young University, Provo, UT 84602 USA (e-mail: long@ee.byu.edu).

Publisher Item Identifier S 0196-2892(00)00017-6. backscatter cross section $\left(\sigma^{\circ}\right)$ at multiple azimuth angles. The geophysical-model function, which relates windspeed and direction to backscatter cross section, is then numerically inverted to infer the near-surface wind. In a significant design departure from previously flown "fan-beam" scatterometer systems, however, SeaWinds is a "pencil-beam" design.

With fan-beam scatterometers such as SASS, NSCAT, and the AMI scatterometer on the European Remote Sensing satellite series (ERS-1 and 2), several fixed antennas are deployed to cast long, narrow illumination patterns at the multiple azimuth angles required for wind retrieval [1], [6], [15]. The narrow dimension of the antenna beam pattern provides resolution in the along-track direction, and Doppler or range filtering is employed to provide cross-track resolution. The antenna structures are typically about three meters in length and require large unobstructed fields-of-view on the spacecraft.

By contrast, planned pencil-beam systems employ a single, approximately $1-\mathrm{m}$ parabolic dish that is conically scanned about the nadir axis to provide multiple azimuth measurements [17], [22] (see Figs. 1 and 2). A key advantage of pencil-beam systems is that, because of their more compact design, they are much easier to accommodate on spacecraft without the necessity of complex deployment schemes or severe field-of-view constraints. In an era in which smaller space missions with faster development times are often mandated (as is the case with the QuikSCAT mission, for example [5]) such a reduction in payload size is highly desirable. An additional advantage of pencil-beam systems is that, because they measure ocean backscatter at a constant incidence angle suitable for wind retrieval, there is no "nadir gap" in swath coverage as there has been for previous fan-beam systems. The resulting contiguous swath offers a significant improvement in Earth coverage. For these reasons, the pencil-beam design has been adopted for SeaWinds and planned follow-on systems into the next century [18].

\section{B. Utility of Higher Resolution Measurements with SeaWinds}

The original design of the SeaWinds instrument, which was developed previous to the flight of NSCAT, is described in [17]. As originally planned, the spatial resolution of the $\sigma^{\circ}$ measurements collected with SeaWinds were to be "beam-limited" (i.e., the dimensions of the $\sigma^{\circ}$ cell are determined by the antenna beam footprint on the surface). For the SeaWinds 1-m antenna, the resulting footprint dimensions are roughly $25 \mathrm{~km} \mathrm{x} 35 \mathrm{~km}$ (see Table I). This resolution approach was selected for two reasons: 1) it satisfied resolution requirements for the primary scatterometer mission to measure synoptic-scale global winds and 


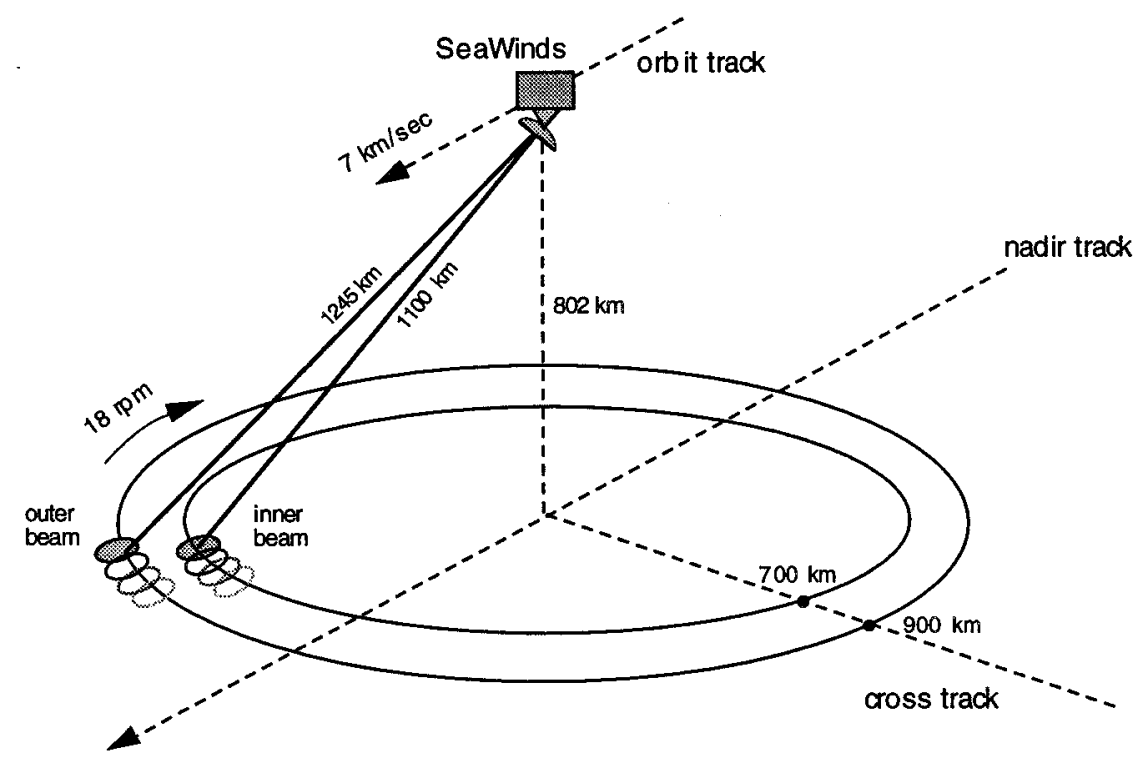

Fig. 1. SeaWinds measurement geometry.

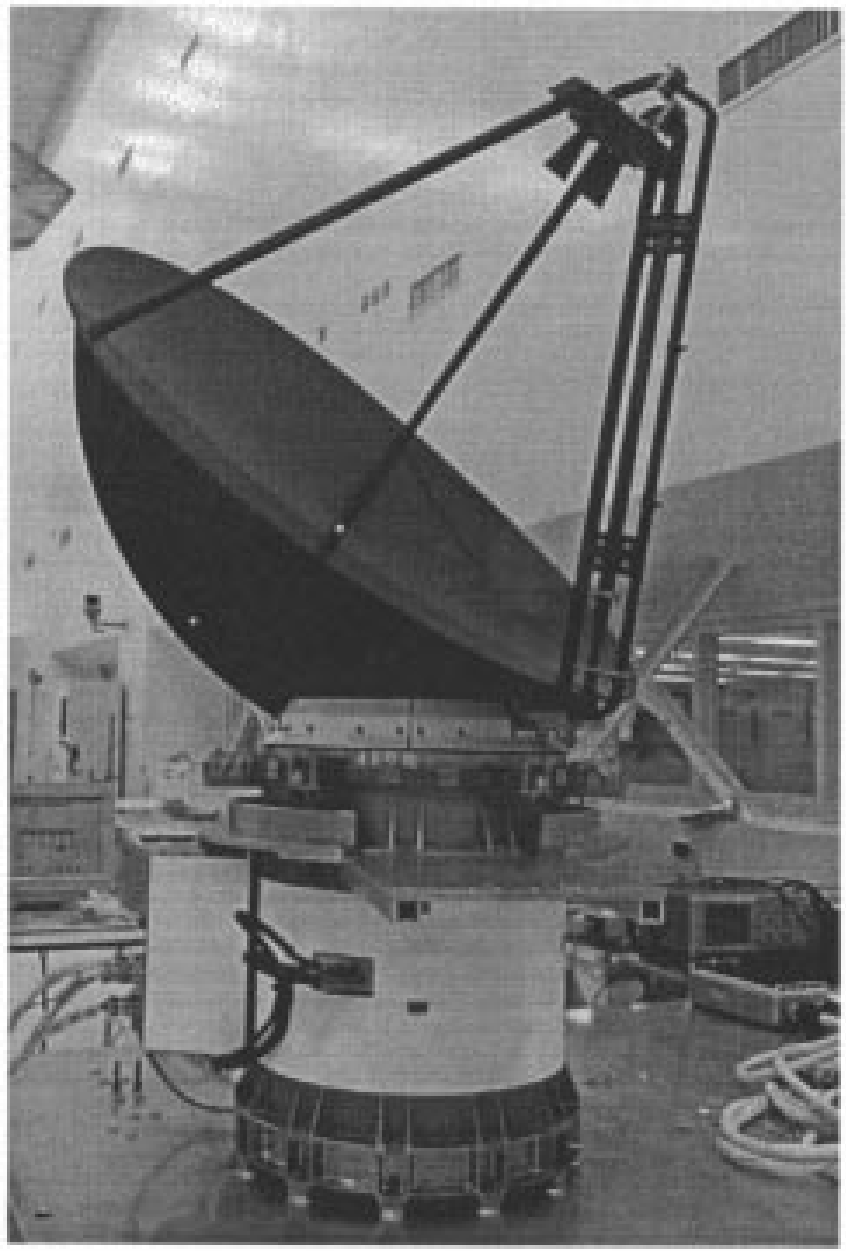

Fig. 2. SeaWinds antenna and rotary mechanism.

2) it enabled high radiometric precision and calibration accuracy to be achieved with a very simple hardware design.

The success of the NSCAT mission, however, demonstrated the benefits of measuring surface backscatter at higher res-
TABLE I

SEAWINDS ANTENNA AND MEASUREMENT-GEOMETRY PARAMETERS

\begin{tabular}{l|c|c}
\hline Parameter & Inner Beam & Outer Beam \\
\hline Polarization & $\mathrm{H}$ & $\mathrm{V}$ \\
Elevation Angle & $40^{\circ}$ & $46^{\circ}$ \\
Surface Incidence Angle & $47^{\circ}$ & $55^{\circ}$ \\
Slant Range & $1100 \mathrm{~km}$. & $1245 \mathrm{~km}$. \\
3 dB Beam Widths $(\mathrm{az} \times \mathrm{el})$ & $1.8^{\circ} \times 1.6^{\circ}$ & $1.7^{\circ} \times 1.4^{\circ}$ \\
Two-Way 3 dB Footprint Dimensions $(\mathrm{az} \times \mathrm{el})$ & $24 \times 31 \mathrm{~km}$ & $26 \times 36 \mathrm{~km}$ \\
Peak Gain & $38.5 \mathrm{dBi}$ & $39 \mathrm{dBi}$ \\
Rotation Rate & \multicolumn{2}{|c}{$18 \mathrm{rpm}$} \\
Along Track Spacing & $22 \mathrm{~km}$. & $22 \mathrm{~km}$. \\
Along Scan Spacing & $15 \mathrm{~km}$. & $19 \mathrm{~km}$. \\
\hline
\end{tabular}

olution. Although NSCAT was also designed primarily as a synoptic wind instrument, the inherent spatial resolution of the backscatter measurements was somewhat higher than that originally planned for SeaWinds. The narrow NSCAT antenna patterns were Doppler filtered to form $\sigma^{\circ}$ "cells" of approximately $8 \mathrm{~km} \mathrm{x} 25 \mathrm{~km}$. These measurements proved to be extremely useful in new scientific applications for spaceborne scatterometry. Wind fields constructed by utilizing the full resolution capability of NSCAT exhibit mesoscale motions in detail [4], [8], allowing more in-depth analysis of storms, frontal zones, orographic effects, and coastal phenomena.

In addition to ocean-wind vector retrieval, scatterometer $\sigma^{\circ}$ measurements are also finding increased applicability in land and ice studies, as surface backscatter can be a sensitive indicator of environmental change (see, for example, [10], [13], and [21]). The utility of the scatterometer data for land and ice studies is significantly expanded by using a technique referred to as enhanced resolution imaging (ERI). With ERI, multiple passes of overlapping scatterometer data over the same region 
are combined to solve for backscatter images that have higher resolution than the original measurements [9]. Essentially, this process is equivalent to a deconvolution of the $\sigma^{\circ}$ measurement spatial-response function. Although images obtained by ERI are still of much lower resolution than that obtainable with synthetic aperture radar (SAR), they have the advantage of very frequent global coverage. Images obtained by ERI have been used in studies of polar ice and the Amazon rain forest [10], [13].

Examples of algorithms that have been successfully employed to achieve resolution enhancement of microwave remote-sensing data include Backus-Gilbert Inversion [16] and scatterometer image reconstruction with filtering (SIRF) [9], [12]. As observed with the SIRF algorithm, the practical resolution achievable with ERI is roughly equivalent to the narrowest dimension of the $\sigma^{\circ}$ measurement cell. For NSCAT, this limiting resolution is approximately $8 \mathrm{~km}$. If beam-limited SeaWinds $\sigma^{\circ}$ cells are used, however, the corresponding enhanced resolution achievable is only about $25 \mathrm{~km}$.

Thus, in order to extend the valuable higher resolution capability demonstrated with NSCAT to the next series of Ku-band scatterometers, the SeaWinds design must be modified to produce backscatter measurements with improved resolution. A challenge accompanying any such modification is that high radiometric precision and calibration accuracy (characteristics that allow the scatterometer to retrieve winds and detect global change) must be preserved.

This paper discusses the various options and tradeoffs considered for improving the resolution of a scanning pencil-beam scatterometer. The specific design modification implemented on SeaWinds for flights on QuikSCAT and ADEOS-II (the addition of range discrimination capability) is described. In Section III, the new challenges associated with calibrating these higher resolution $\sigma^{\circ}$ 's are addressed, and the radar equation for a rotating pencil-beam scatterometer with range filtering is presented. Also in Section III, the issue of measurement precision is addressed, and a formulation for the measurement variance for the higher resolution $\sigma^{\circ}$ 's is given. Finally, in Section IV, simulation results are shown that demonstrate the enhanced backscatter imaging capability achievable with the modified system relative to what was originally achievable with the beam-limited system. Although the presentation here is primarily directed toward describing the SeaWinds system, the principles developed are applicable to future scanning pencil-beam scatterometer designs.

\section{IMPROVED RESOLUTION APPROACH}

\section{A. Range Versus Doppler Discrimination}

Before describing the specific range-discrimination modification implemented on SeaWinds, it is insightful to discuss the reasons for selecting this approach over other resolution-improvement options. The overriding consideration was that because the modifications were to be made late in the hardware development, only a minimum of changes to the overall instrument architecture could be accommodated. Furthermore, the ability to achieve high radiometric accuracy and adequate Earth coverage could not be compromised. In general, these constraints implied only changes to the radar modulation and signal-processing electronics, with no changes to the spacecraft orbit, antenna subsystem, or transmitter, and with only a modest increase in data rate.

With these limitations in mind, it is instructive to consider the overall geometry and range/Doppler characteristics of the SeaWinds backscatter measurement. As described in [17], SeaWinds employs a 1-m diameter dish antenna with offset feeds to generate two pencil-beams (the "inner" beam at an off-nadir angle of $40^{\circ}$ and the "outer" beam at an off-nadir angle of $46^{\circ}$; see Fig. 1). The antenna is then conically scanned such that each point on the Earth within the inner $700 \mathrm{~km}$ of the swath is viewed from four different azimuth directions. They are viewed twice by the inner beam looking forward then aft and twice by the outer beam in a similar fashion. Other relevant parameters for the antenna and scan geometry are given in Table I.

The approximate dimensions of the antenna two-way 3-dB footprint contour, along with the associated two-way iso-range and iso-Doppler lines are conceptually illustrated in Fig. 3. Two representative cases are shown. One is the case in which the beam is scanned to an azimuth angle of $0^{\circ}$ (beam looking in the direction of spacecraft motion), and the other is the case in which the beam is scanned to an azimuth of $90^{\circ}$ (beam looking perpendicular to spacecraft motion). Note that for the $0^{\circ}$ azimuth case, the iso-range and iso-Doppler lines are approximately parallel, whereas in the $90^{\circ}$ case, they are nearly perpendicular. Other azimuth angles will yield various intermediate states of these two cases, with the range and Doppler lines slanting with respect to each other.

Ideally, we desire a processing scheme that resolves the footprint in two orthogonal dimensions simultaneously, in effect obtaining a low-resolution SAR measurement with both range and Doppler resolution. Unfortunately, this goal is problematic with the existing SeaWinds architecture. In addition to increased processor complexity, one reason for this is that the diameter of the SeaWinds antenna $(1 \mathrm{~m})$ is smaller than that required to simultaneously avoid range and Doppler ambiguities [20]. To see this, consider that the beam fill time (the difference in roundtrip flight time from the near edge to the far edge of the antenna footprint) is approximately $0.28 \mathrm{~ms}$. To avoid range ambiguities within the footprint, this implies a maximum PRF of 3.6 $\mathrm{kHz}$. The total Doppler bandwidth across the footprint, however, is approximately $10 \mathrm{kHz}$, and thus requires a minimum 10 $\mathrm{kHz}$ PRF in order to unambiguously resolve the scene in azimuth. A measurement without ambiguity difficulties would require an antenna diameter in excess of two meters to achieve a sufficiently narrow beamwidth, violating a key requirement that the instrument be physically compact. Even if the antenna were large enough, as the antenna is scanned near $0^{\circ}$ or $180^{\circ}$ azimuth, the Doppler and range lines are nearly parallel, degrading the two-dimensional (2-D) resolution.

As an alternative, we consider performing either pure range filtering or pure Doppler filtering to achieve spatial "slices" through the antenna footprint, where resolution is only improved in one dimension. Again referring to Fig. 3, we have illustrated idealized slices (or "cells," as they are also called) formed by range discrimination or Doppler discrimination with dark-shaded and light-shaded regions, respectively. As 
Forward Scan $(A z=0$ deg $)$

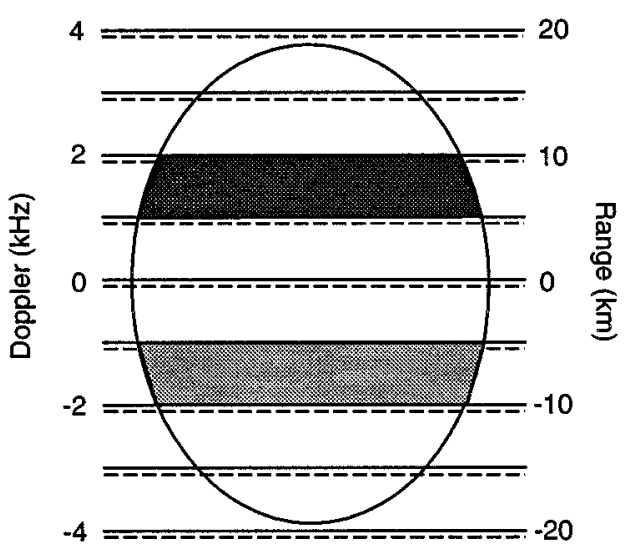

Side Scan $(A z=90 \mathrm{deg})$

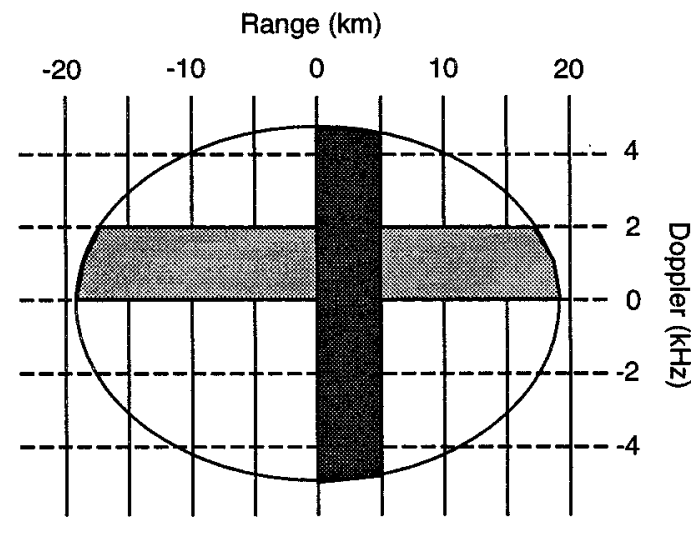

Fig. 3. Conceptual diagram of SeaWinds 3-dB antenna footprint projected on the surface (oval) with two-way iso-range (solid) and iso-Doppler (dashed) lines. Shaded areas illustrate range-filtered (dark) and Doppler-filtered (light) resolution elements.

will be demonstrated more fully in Section IV, the fact that we can improve resolution in at least one dimension is still of significant benefit, particularly for land and ice images constructed with ERI. To decide whether range or Doppler filtering should be employed for SeaWinds, two issues were considered: backscatter measurement variance and the geometrical orientation of the resultant $\sigma^{\circ}$ cells.

The measurement-variance issue is discussed in more detail in Section III, but a brief argument is given here to justify the filtering strategy selected for SeaWinds. Scatterometer measurements of surface $\sigma^{\circ}$ are noisy due to Rayleigh fading and because of the presence of thermal noise. A key goal of scatterometer design is to minimize the $\sigma^{\circ}$ noise variance. When the SNR is large, as it generally is for land, ice, and moderate to high ocean winds, Rayleigh fading is the dominant factor, and the variance of each $\sigma^{\circ}$ measurement is related to the number of independent "looks" achieved [20].

In the Doppler-filtering case, the maximum number of "looks" available is related to the Doppler frequency resolution associated with the transmit signal. For the selected SeaWinds timing, the maximum integration time on each scene is 1.5 $\mathrm{ms}$, implying a best Doppler resolution of $1 /(1.5 \mathrm{~ms})=666$ $\mathrm{Hz}$. Given that the total Doppler bandwidth of the illuminated region is about $10 \mathrm{kHz}$, the footprint could theoretically be resolved into as many as $10000 \div 666=15$ separate elements, each constituting one independent "look" at the surface. If the footprint is equally divided into four resolution slices, each slice would thus contain the equivalent of 3.75 independent looks, corresponding to a normalized-measurement standard deviation of $52 \%(1 / \sqrt{3.75}=0.52)$ of the actual $\sigma^{\circ}$ value. Measurement variance cannot be further reduced without improving the Doppler resolution by lengthening the transmit pulse, which is not allowed by the timing constraints.

In the range-filtering case, however, the inherent resolution is a function of the bandwidth of the modulated transmit pulse. If the transmit pulse is modulated with a linear chirp at a rate of $250 \mathrm{kHz} / \mathrm{ms}$, the resulting pulse will have a bandwidth of 375 $\mathrm{kHz}$, corresponding to a time-delay resolution of $1 /(375 \mathrm{kHz})$ $=2.7 \mu \mathrm{s}$. This in turn corresponds to a surface-distance reso- lution of about $0.7 \mathrm{~km}$ for the outer beam. If the $36 \mathrm{~km}$-long outer-beam footprint is divided equally into four $9 \mathrm{~km}$ slices, this implies $9 \div 0.7=12.86$ looks per slice, or a measurement standard deviation of $28 \%(1 / \sqrt{12.86}=0.28)$ of the true $\sigma^{\circ}$ value. If SNR is sufficiently high, the measurement variance can be further improved in the range-filtering case by increasing the bandwidth of the transmitted pulse. The flexibility to improve the measurement accuracy of the slices, and consequently the accuracy of geophysical products such as winds and surface images, by adjusting the transmit modulation bandwidth is a key advantage of the range-filtering approach.

A rough, intuitive assessment of the comparative wind performance for different modulation approaches may be made by considering the variance when all backscatter measurements are combined in the wind retrieval. For wind estimates at $25-\mathrm{km}$ resolution, all $\sigma^{\circ}$ slices from all azimuth directions which fall in a $25 \mathrm{~km}$ box or "wind vector cell" on the ocean surface are used in the wind retrieval. If each footprint is divided into four slices, the SeaWinds PRF and scan rate dictates that there will be, on average, about 40 slices available for each wind measurement. In a simplified sense, the windspeed accuracy achievable can be estimated by considering the standard deviation that results when all $40 \sigma^{\circ}$ measurements are averaged. For the Doppler resolution case discussed above, the effective combined standard deviation is thus $8 \%(1 / \sqrt{40 \times 3.75}=0.08)$ or, equivalently, $0.33 \mathrm{~dB}$ of the true value. For the range-resolution case, the corresponding standard deviation is $4 \%(1 / \sqrt{40 \times 12.86}=0.04)$ or $0.17 \mathrm{~dB}$ about the actual value. Measurements of high wind speed are most sensitive to errors in $\sigma^{\circ}$. For a $20 \mathrm{~m} / \mathrm{s}$ wind, a $0.33 \mathrm{~dB}$ or $0.17 \mathrm{~dB}$ backscatter error translates into a $1.7 \mathrm{~m} / \mathrm{s}$ or $0.9 \mathrm{~m} / \mathrm{s}$ windspeed error, respectively. Thus, the use of range filtering has the potential to improve wind performance by at least a factor of two over Doppler filtering for the SeaWinds design.

A secondary consideration is the orientation of the $\sigma^{\circ}$ slices. Because ERI algorithms utilize many overlapping $\sigma^{\circ}$ measurements, possibly from multiple orbits, it is generally desirable to have the slices oriented at different angles so that resolution may be enhanced effectively in all directions. As shown in Fig. 3, this requirement favors range filtering because the ori- 


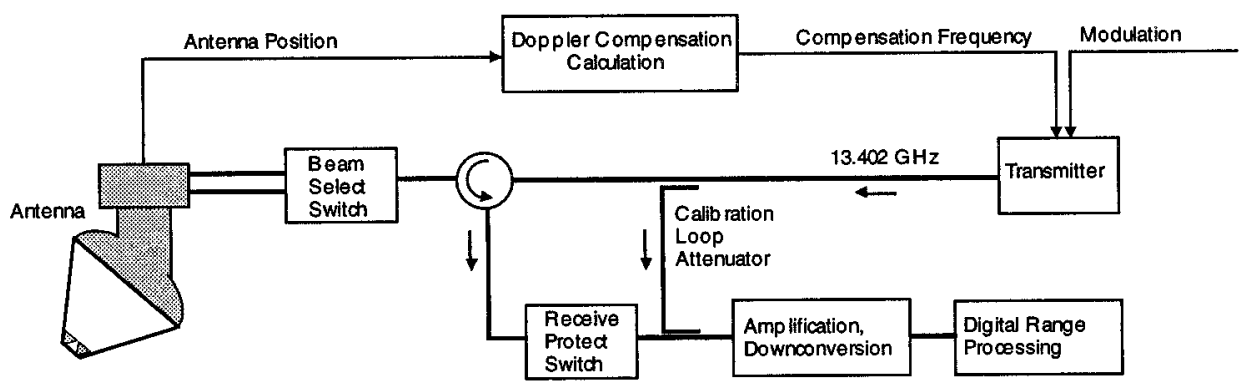

Fig. 4. Block diagram of SeaWinds radar system.

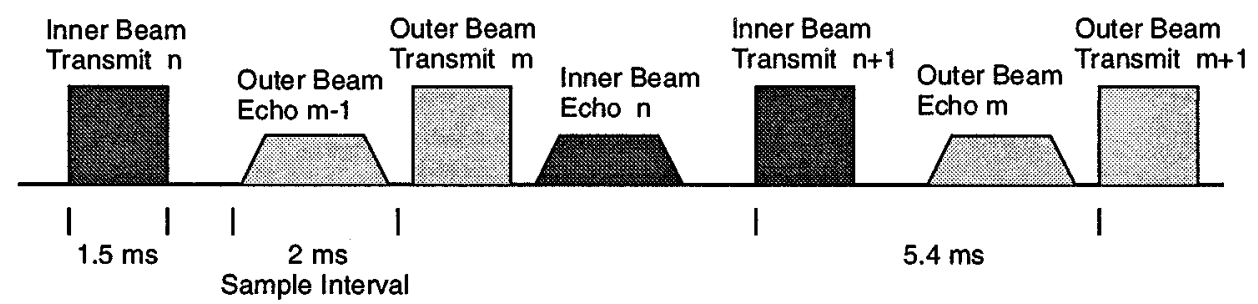

Fig. 5. SeaWinds transmit and receive timing. Note that pulses alternate between inner and outer beam, with two pulses in flight.

entation of the cells rotates with azimuth angle, as opposed to Doppler filtering, in which the cells are oriented roughly perpendicular with the direction of flight. Because of the above-described advantages in measurement variance and cell orientation, the range-discrimination approach was chosen to form the SeaWinds improved-resolution cells.

\section{B. Range-Filtering Implementation}

Because of the relatively low peak power available with the SeaWinds transmitter, a chirp/deramp technique was selected to achieve range resolution. This type of processing is similar to that employed by other spaceborne radar instruments (e.g., [23]), and minimizes modifications to the existing low-resolution SeaWinds design. A functional diagram of the SeaWinds radar is shown in Fig. 4. Upon command from the timing controller, the transmitter, which consists of a modulated signal generator driving a traveling wave tube (TWT) amplifier, issues a 1.5-ms duration, 110-W Ku-band pulse. In the previous design, the pulse was MSK modulated to optimize the measurement variance for the simple "nonmatched" square-law detection scheme used to obtain beam-limited $\sigma^{\circ}$ cells [11], [17]. For range discrimination, the transmit pulse is now modulated with a linear-frequency chirp at a chirp rate of $250 \mathrm{kHz} / \mathrm{ms}$ for a total bandwidth of $375 \mathrm{kHz}$. (A discussion of how this specific chirp rate was selected is in Section III-B.)

Due to the motion of the satellite relative to the Earth, a gross Doppler shift of between $\pm 500 \mathrm{kHz}$ is imparted to the echo return, depending on the antenna-azimuth position. In the SeaWinds design, this Doppler shift is precompensated by tuning the transmit carrier frequency to $13.402 \mathrm{GHz}$ minus the expected Doppler shift from the footprint center location. Precompensating for Doppler shift produces an echo signal that always occurs at the same baseband frequency after downconversion. The transmit pulse is routed to either the inner or outer beam through a coaxial rotary joint to the spinning section of the an- tenna assembly. An important feature of any scatterometer is the accurate calibration of the transmit power and receiver gain (see Section III-A). These parameters are measured simultaneously by periodically injecting the transmit pulse, attenuated by a known amount, into the receiver via the calibration-loop coupler.

The pulse repetition and echo-gate timing, which is designed to provide a sufficiently dense sampling of $\sigma^{\circ}$ measurements on the surface as the antenna rotates, is shown in Fig. 5. Transmit pulses occur every $5.4 \mathrm{~ms}$ and alternate between the inner and outer beams. This produces an effective PRF of $92.5 \mathrm{~Hz}$ for each beam. The roundtrip flight times for the inner and outer beam pulses is approximately $7.3 \mathrm{~ms}$ and $8.3 \mathrm{~ms}$, respectively, and thus, each echo returns after the succeeding transmit pulse. In Fig. 5, the echo returns are depicted as trapezoids to indicate dispersion due to the antenna footprint fill time of approximately $0.3 \mathrm{~ms}$.

The echo signal processing, which constitutes the most significant modification made to implement range discrimination, is diagrammed in Fig. 6. After downconversion to baseband, the echo is digitally sampled. The total echo return, which is the sum of all the echo returns from scatterers across the illuminated region, is then digitally "deramped" by mixing with a chirped reference single. This operation effectively converts range delays into frequency shifts (i.e., each discrete frequency in the deramped signal corresponds to the return from a given range line on the surface plus a small Doppler shift effect discussed in Section III). To extract the range information, a discrete Fourier transform (implemented as an FFT) is performed on the deramped signal and a periodogram is formed by applying a magnitude-squared operation. The periodogram bins are then summed into twelve range-slice energy measurements to be telemetered to the ground.

To illustrate further, Fig. 7 shows a conceptual plot of the deramped power spectral density (shaded region) and slice band- 


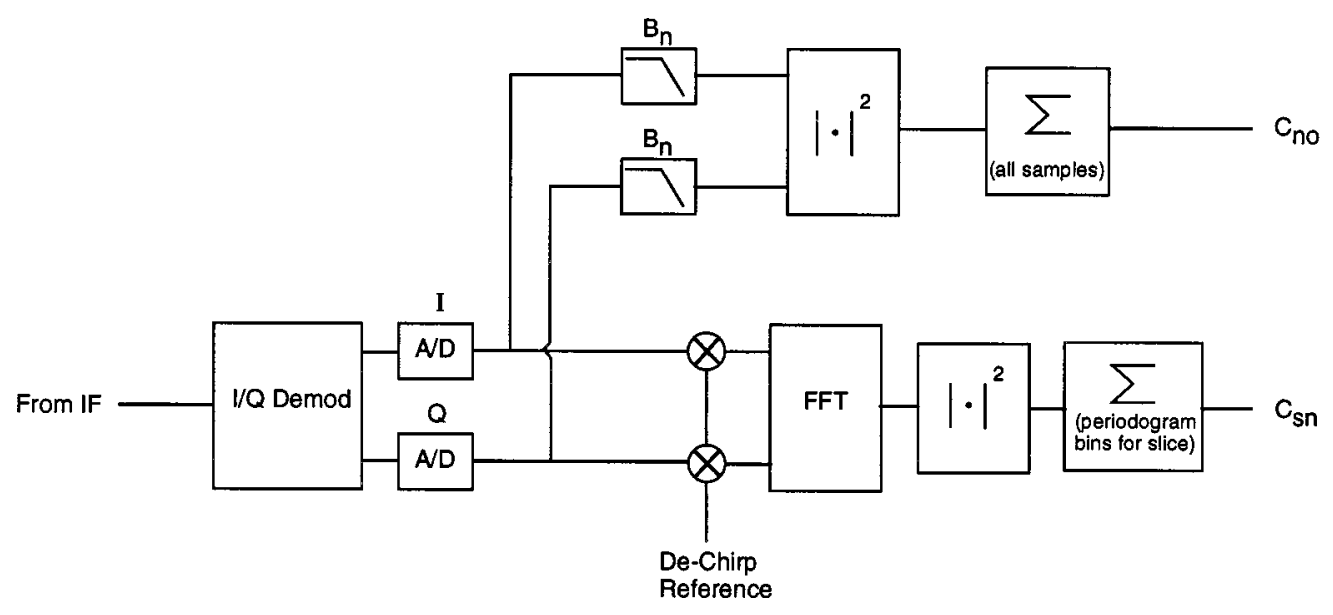

Fig. 6. Functional diagram of SeaWinds digital-range processing and "noise-only" processing.

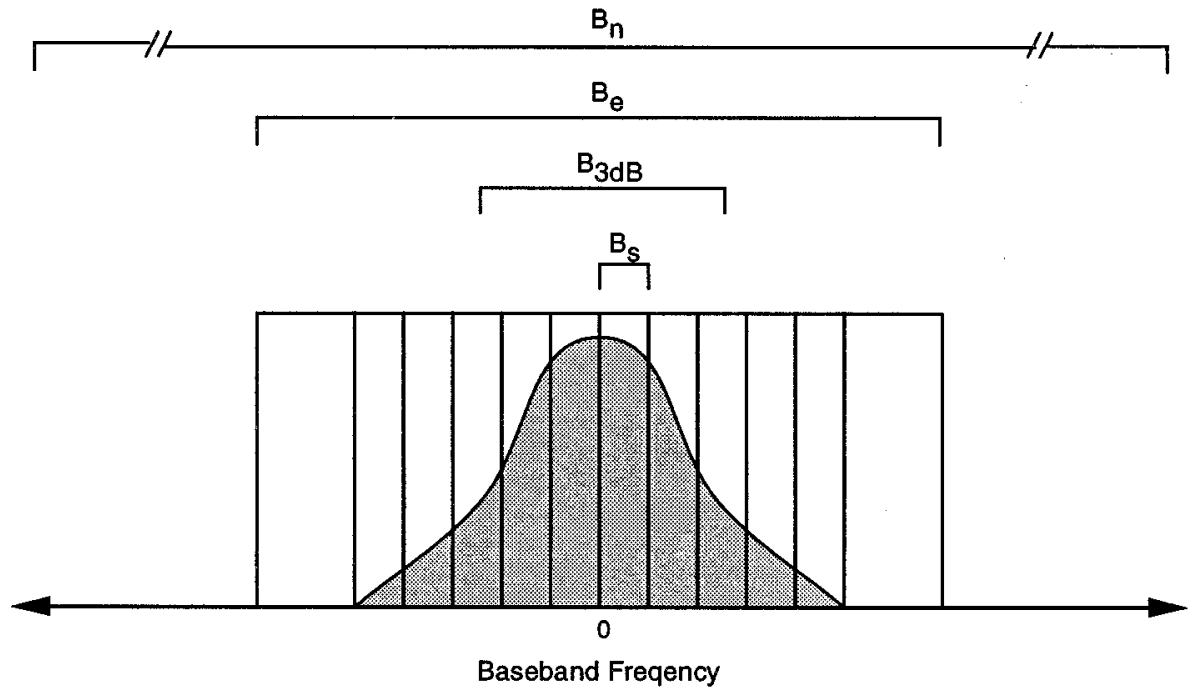

Fig. 7. Conceptual diagram illustrating deramped echo spectrum (shaded), range slices, and other bandwidths used in processing. The energy in each of the 12 spectral slices is accumulated to form each measurement $C_{s \eta n}^{q}$.

widths. For the selected SeaWinds chirp rate of $250 \mathrm{kHz} / \mathrm{ms}$, the deramped spectral density has a $3-\mathrm{dB}$ bandwidth $\left(B_{3 \mathrm{~dB}}\right)$ of approximately $40 \mathrm{kHz}$. The returned energy for the $q$ th slice $C_{s n}^{q}$ is formed by summing adjacent periodogram bins over the slice bandwidth $B_{s}$. For SeaWinds, it was decided that $\sigma^{\circ}$ cells that resolve the surface to approximately $7 \mathrm{~km}$ in the narrow (range) dimension would be constructed. For the given chirp rate, this corresponds to $B_{s}=8.3 \mathrm{kHz}$. This bandwidth is used for the ten innermost slices. The two outermost slices are termed "guard slices," and are assigned a somewhat larger bandwidth. The total bandwidth spanned by all twelve slices $B_{e}$ is approximately 200 $\mathrm{kHz}$, and is designed so as to capture the entire deramped echo spectrum.

As in the previous design [17], a wideband "noise-only" measurement $\left(C_{n o}\right)$ is made by passing the return echo and system noise through a filter with bandwidth $B_{n}=1 \mathrm{MHz}$, then square-law detecting, and then integrating. This measurement is used in determining the thermal-noise background component to $C_{s n}^{q}$, which must be subtracted off before $\sigma^{o}$ can be estimated.

\section{C. $\sigma^{\circ}$ Estimation}

The estimation of $\sigma^{\circ}$ from the telemetered measurements is essentially a two-step process. First, an estimate of the thermal noise contribution (instrument plus Earth scene) to a given slice $P_{n}^{q}$ must be subtracted from the slice measurement $C_{s n}^{q}$ to yield an estimate of the "signal-only" power $P_{s}^{q}$

$$
P_{s}^{q}=C_{s n}^{q}-P_{n}^{q} .
$$

For low windspeeds, the noise contribution may be ten times the signal strength, and thus, the accurate determination of $P_{n}^{q}$ is a crucial step (see the Appendix).

The second step is to relate the signal-only echo energy to a value of $\sigma^{\circ}$ on the Earth's surface by applying the radar calibration parameter $X$

$$
\sigma^{\circ}=\frac{P_{s}^{q}}{X}
$$

Equation (2) is a shorthand expression for the distributed-target radar equation [20]. Here, $X$ incorporates all instrumental 
and geometrical parameters (antenna gain, transmit power, slant range, etc.) necessary to define the relationship between detected echo energy and $\sigma^{\circ}$. The radar equation specific to a scanning pencil-beam scatterometer with digital-range filtering is developed in the next section.

\section{BACKSCATTER MEASUREMENT ACCURACY}

In the previous section, the overall resolution approach, along with the selected signal-processing implementation, was described. Although it is clear that resolution is easily enhanced by the addition of range filtering, it is critical that the issue of $\sigma^{\circ}$-measurement accuracy be addressed as well. A fundamental requirement of scatterometer instruments is the ability to measure surface backscatter with very high accuracy. Such accuracy is required to retrieve winds and detect long-term climatic change. In general, achieving the desired measurement accuracy is more difficult with $\sigma^{\circ}$ cells formed by "range slicing" than in the simple beam-limited case and requires the introduction of new formulations for the radar equation and backscatter measurement variance calculation. This section provides the necessary analytical framework for addressing the issue of $\sigma^{\circ}$ accuracy for SeaWinds with digital range filtering and discusses several of the tradeoffs that must be conducted to optimize performance. We address two main aspects of measurement accuracy: $\sigma^{\circ}$ calibration associated with the $X$ parameter in (2) and $\sigma^{\circ}$-measurement variance associated with random fluctuations in $P_{s}^{q}$ due to fading and thermal noise.

\section{A. $\sigma^{\circ}$ Calibration}

Calibration accuracy refers to the scatterometer's ability to determine the true backscatter cross section given the intensity of the echo return. In essence, it is the accuracy with which we know the radar parameter $X$ in (2). In general, calibration error can be divided into two sources: radiometric errors, which are caused by uncertainty in our knowledge of instrument-component gains and losses, and geometric errors, which arise chiefly from imperfect knowledge of the exact pointing of the antenna pattern. As will be discussed, it is primarily the geometric errors that limit the calibration performance of a well-designed scatterometer instrument.

It is also important to differentiate between "absolute" and "relative" calibration errors. An absolute error is the degree to which a measurement of $\sigma^{\circ}$ differs from the "true" value. A relative calibration error, however, is the difference in absolute error between two $\sigma^{\circ}$ measurements separated in space and/or time. Because we are most interested in detecting either spatial or temporal change in surface backscatter, it is particularly important to minimize relative error. To produce marine wind fields with the desired accuracy and to allow maximum sensitivity to climatologically induced changes in surface backscatter, relative calibration accuracy of $0.2 \mathrm{~dB}$ is desired. This challenging goal requires a detailed consideration of all radiometric and geometric factors affecting the $X$ parameter for each $\sigma^{\circ}$ cell.

1) Expression for $X$ : The first step in the calibration of the instrument involves the development of an expression for the parameter $X$. In deriving $X$, we track the radar signal through its interaction with the surface and the subsequent echo signal processing.

The transmitted signal can be written as

$$
T(t)=\sqrt{E_{t}} p(t) e^{j 2 \pi\left[f_{c}+f_{d c}+(1 / 2) \mu t\right] t}
$$

where $t$ is time from onset of transmit pulse, $E_{t}$ is total energy in transmit pulse, $p(t)$ is the transmit pulse-power envelope such that $\int p^{2}(t)=1, f_{c}$ is the transmit-carrier frequency, $f_{d c}$ is the Doppler-compensation frequency, and $\mu$ is the chirp rate.

The echo return from the surface can be treated as the summation of returns from many independent scattering "patches," each with a different range delay and Doppler shift [11], [20]. It is assumed that each patch is large relative to the correlation length of the surface but sufficiently small so that the Doppler shift and slant range do not vary significantly over its dimensions. The echo return from the $i$ th scattering patch is expressed as

$$
\begin{aligned}
R_{i}(t)= & \xi_{i} \sqrt{\sigma_{i}^{O}} C B_{i}(t) \\
& \cdot e^{j 2 \pi\left\{\left[f_{c}+f_{d c}+f_{d, i}+(1 / 2) \mu\left(t-t_{d, i}\right)\right]\left(t-t_{d, i}\right)+\phi_{i}\right\}} .
\end{aligned}
$$

Here, $\sigma_{i}^{o}$ is the normalized backscatter cross section at the scattering patch, $\xi_{i}$ is a univariant Rayleigh random variable for the signal amplitude due to fading, $\phi_{i}$ is a uniform random variable (over $0-2 \pi$ ) for the random phase of the return from the patch, $f_{d, i}$ is the Doppler shift of the patch, and $t_{d, i}$ is the roundtrip flight time to the patch given by $t_{d, i}=2 r_{i} / c$ where $r_{i}$ is the slant range to the patch.

The value $C$ in (4) is defined such that

$$
C^{2}=\left(\frac{\lambda^{2}}{(4 \pi)^{3}}\right)\left(\frac{E_{t} G_{r} G_{p}^{2}}{L_{s y s}}\right)
$$

where $\lambda$ is the transmit wavelength, $G_{r}$ is the receiver gain, $G_{p}$ is the peak antenna gain, and $L_{s y s}$ is the total two-way system loss. The echo return is windowed by the function $B_{i}(t)$

$$
B_{i}(t)=p\left(t-t_{d, i}\right)\left(\frac{\delta A_{i}}{r_{i}^{4}}\right)^{1 / 2}\left[g_{i}(t) g_{i}\left(t-t_{d, i}\right)\right]^{1 / 2}
$$

where $\delta A_{i}$ is the area of the scattering patch, and $g_{i}(t)$ is the normalized antenna-pattern gain in the direction of the $i$ th scattering patch at time $t$. The term $g_{i}(t) g_{i}\left(t-t_{d, i}\right)$ reflects the fact that the antenna gain is changing as a function of time as the antenna rotates (see [14]).

The composite return over the entire footprint $R(t)$ is given by

$$
R(t)=\sum_{i \in \mathcal{F}} R_{i}(t)
$$

where the summation is over all contiguous, unique patches in the illuminated region $\mathcal{F}$. In this analysis, discrete summation over the illuminated region, rather than the more conventional integral representation, is used for clarity and to reflect the fact that $X$ is evaluated numerically in practice. As with all numerical integrations, the size of $\delta A_{i}$ and the extent of $\mathcal{F}$ are ultimately selected such that acceptable convergence is achieved. 
At the receiver, the signal is downconverted and deramped by multiplying $R_{i}(t)$ with

$$
M(t)=\exp \left\{-\pi \mu j\left(t-t_{g}\right)\left(t-t_{g}\right)\right\}
$$

where $t_{g}$ is the reference delay, to yield

$$
\begin{aligned}
R_{d}(t) & =R(t) M(t) \\
& =C \sum_{i \in \mathcal{F}} \xi_{i} \sqrt{\sigma_{i}^{o}} B_{i}(t) \exp \left\{2 \pi j f_{b, i} t+j \psi_{i}\right\} .
\end{aligned}
$$

Here, $f_{b, i}$ is the baseband frequency of the return from the $i$ th patch and is given by

$$
f_{b, i}=f_{d, i}+f_{d c}+\mu\left(t_{g}-t_{d, i}\right) .
$$

The new phase term $\psi_{i}$ is a function of $\phi_{i}$ but is still a random variable uniformly distributed over $(0-2 \pi)$. Examining (9) and (10), it is evident that the deramped echo is a composite of many scaled, windowed, single-frequency tones with random phase. Each tonal frequency is determined by the range delay and Doppler shift associated with each scatterer. Note then, that the processing does not represent pure range filtering because the iso-baseband frequency lines on the surface will be somewhat tilted with respect to the iso-range lines, the magnitude of the tilt being a function of the chirp rate $\mu$.

The deramped signal is then digitally sampled and gated to form the sequence $d[n]$

$$
d[n]=C \sum_{i \in \mathcal{F}} \xi_{i} \sqrt{\sigma_{i}^{\circ}} G\left(t_{n}\right) B_{i}\left(t_{n}\right) \exp \left\{2 \pi j f_{b, i}\left(t_{n}\right)+j \psi_{i}\right\} .
$$

Here, the sample time $t_{n}$ is equal to $t_{g s}+n T$, where $t_{g s}$ is the time associated with the first sample input to the DFT, $n$ is the sample number, and $T$ is the sample period. $G(t)$ is a rectangular window function representing the range gate ( $G=1$ for signal "on" or $G=0$ for signal "off").

To form the slice measurement $P_{s}^{q}$, a DFT is applied to the sample sequence, the Fourier domain sequence is magnitude squared, and then the appropriate periodogram bins are summed. Also, we assume that the backscatter cross section is constant over the slice to obtain

$$
\begin{aligned}
P_{s}^{q}= & \sigma^{\circ} C^{2} \sum_{k=k_{s}}^{k_{e}} \mid \sum_{n=0}^{N-1}\left[\sum_{i \in \mathcal{F}} \xi_{i} G\left(t_{n}\right) B_{i}\left(t_{n}\right)\right. \\
& \left.\cdot \exp \left\{2 \pi j f_{b, i}\left(t_{n}\right)+j \psi_{i}\right\}\right]\left.\cdot \exp \left\{-2 \pi j \frac{k n}{N}\right\}\right|^{2}
\end{aligned}
$$

where $k_{s}$ and $k_{e}$ are the start and end bin numbers (corresponding to the bounding frequencies from Fig. 7) of the $q$ th slice, and $N$ is the total number of samples input to the DFT (i.e., FFT size).

Because $P_{s}^{q}$ is a random quantity, we must take the expected value to find $X$

$$
\begin{aligned}
\mathcal{E}\left[P_{s}^{q}\right]= & \sigma^{\circ} C^{2} \sum_{k=k_{s}}^{k_{e}} \sum_{i \in \mathcal{F}} \mid \sum_{n=0}^{N-1} G\left(t_{n}\right) B_{i}\left(t_{n}\right) \\
& \left.\cdot \exp \left\{2 \pi j\left(f_{b, i} T-\frac{k}{N}\right) n\right\}\right|^{2}
\end{aligned}
$$

where, to eliminate the random variables and reorder the summations, we have utilized the assumption that separate scattering patches are uncorrelated (i.e., that $\mathcal{E}\left[e^{j \psi_{m}} e^{-j \psi_{n}}\right]=0$ for $m \neq n$ ), and that $\xi$ is univariant. Referring to (2), we conclude that

$$
\begin{aligned}
X= & C^{2} \sum_{k=k_{s}}^{k_{e}} \sum_{i \in \mathcal{F}} \mid \sum_{n=0}^{N-1} G\left(t_{n}\right) B_{i}\left(t_{n}\right) \\
& \left.\cdot \exp \left\{2 \pi j\left(f_{b, i} T-\frac{k}{N}\right) n\right\}\right|^{2} .
\end{aligned}
$$

Equation (14) is a general expression for $X$ for a rotating antenna with digital-range filtering.

Under certain conditions (which apply in the case of SeaWinds), the computation of $X$ may be simplified somewhat. If the transmit pulse envelope is rectangular, we can write

$$
\begin{aligned}
& p(t)=\frac{1}{\sqrt{T_{p}}}, \quad \text { for } t_{p s} \leq t<t_{p s}+T_{p} \\
& p(t)=0, \\
& \text { otherwise }
\end{aligned}
$$

where $t_{p s}$ is the time of transmit-pulse start. If we further assume that the antenna gain in the direction of a given surface patch is constant during the pulse length, we can also write

$$
B_{i}\left(t_{n}\right)=p\left(t_{n}-t_{d, i}\right)\left(\frac{\delta A_{i}}{r_{i}^{4}}\right)^{1 / 2}\left[g_{i}\left(t_{t r s}\right) g_{i}\left(t_{r e c}\right)\right]^{1 / 2}
$$

where $g_{i}\left(t_{t r s}\right)$ is the gain in the direction of the $i$ th patch at the time of transmit, and $g_{i}\left(t_{r e c}\right)$ is the gain in the direction of the $i$ th patch at the time of receive after the antenna has rotated during the pulse roundtrip flight time $t_{d, i}$. The previous two assumptions are equivalent to assuming that the echo return from a given scatterer is flat and is not modulated by either the pulse envelope or the rotating antenna beam. The sampled signal values will thus correspond to a rectangular window whose length is determined by the overlap between the delayed return pulse and the range-gate window.

Employing (15) and (16), (14) can be written as

$$
\begin{aligned}
X= & \frac{C^{2}}{T_{p}} \sum_{k=k_{s}}^{k_{\epsilon}} \sum_{i \in \mathcal{F}}\left(\frac{\delta A_{i} g_{i}\left(t_{\text {trs }}\right) g_{i}\left(t_{\text {rec }}\right)}{r_{i}^{4}}\right) \\
& \cdot\left|\sum_{n=n_{s, i}}^{n_{s, i}+N_{p, i}} \exp \left\{2 \pi j\left(f_{b, i} T-\frac{k}{N}\right) n\right\}\right|^{2} .
\end{aligned}
$$

Here, $n_{s, i}$ is the sample in the sequence $d[n]$ associated with the leading edge of the gated echo from the $i$ th patch. $N_{p, i}$ is the length of the echo from the $i$ th patch (given in number of samples) captured by the range gate. $N_{p, i}$ is given by

$$
N_{p, i}=\operatorname{int}\left(T_{p} \sum_{n=0}^{N-1} p_{r}\left(t_{n}-t_{d, i}\right) G\left(t_{n}\right)\right) .
$$

It will be convenient, particularly for the analysis of measurement variance, to define the DFT term in (17) as the function $\beta$ where

$$
\beta(i, k)=\sum_{n=n_{s, i}}^{n_{s, i}+N_{p, i}} \exp \left\{2 \pi j\left(f_{b, i} T-\frac{k}{N}\right) n\right\} .
$$




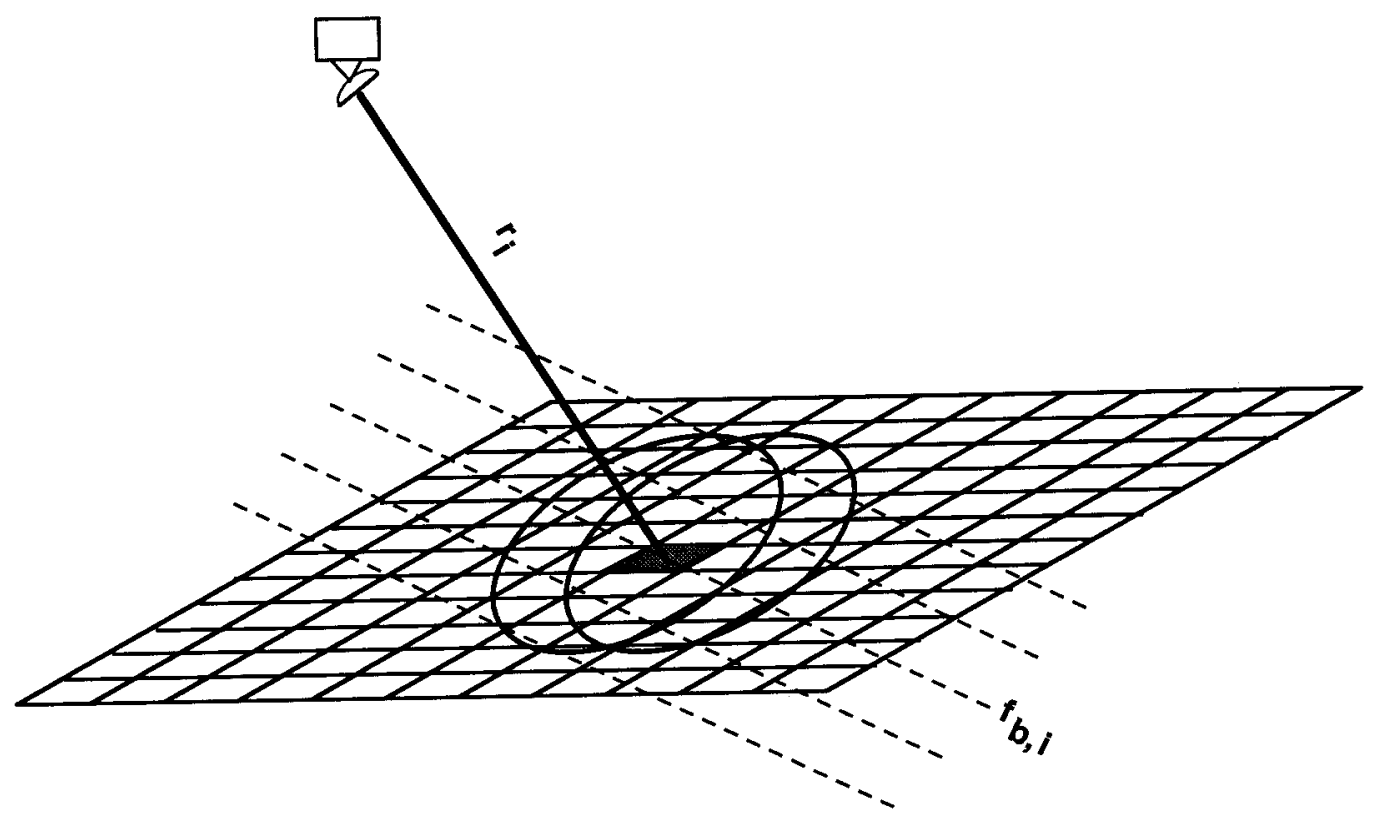

Fig. 8. $X$-parameter calculation geometry. Shaded region represents the $i$ th surface element with incremental area $\delta A_{i}$. Dotted lines represent lines of constant baseband frequency, with baseband frequency of $i$ th element $f_{b}, i$. Offset ovals represent antenna-footprint position at the time of transmit and receive. Range from the $i$ th element to sensor is $r_{i}$.

Evaluating (19), we find that

$$
\begin{aligned}
\beta(i, k)= & \left(\frac{\exp \left[j \pi\left(N_{p, i}+2 n_{s, i}\right)\left(f_{b, i} T-\frac{k}{N}\right)\right]}{\exp \left[j \pi\left(f_{b, i} T-\frac{k}{N}\right)\right]}\right) \\
& \cdot\left(\frac{\sin \left[\pi N_{p, i}\left(f_{b, i} T-\frac{k}{N}\right)\right]}{\sin \left[\pi\left(f_{b, i} T-\frac{k}{N}\right)\right]}\right) .
\end{aligned}
$$

Note that because the complex phase term in $|\beta(i, k)|^{2}$ cancels, we can always sum from 0 to $N_{p, i}$ in the DFT, regardless of the pulse position in the range gate. Equation (17) becomes (21), as shown at the bottom of the page.

For clarity, Fig. 8 is provided to illustrate the integration over the scattering patches. Using (21), $X$ for a sample slice is plotted versus orbit position and antenna azimuth angle for the expected QuikSCAT orbit in Fig. 9. Note that the value of $X$ varies significantly, necessitating an adjustment as a function of orbit position and antenna azimuth to maintain calibration accuracy. Despite the simplifications embodied in (21), $X$ is still too computationally expensive to compute repeatedly for each individual pulse during ground-data processing. Where the satellite orbit is very stable, as is expected for both the QuikSCAT and ADEOS-II spacecraft, $X$ may be precomputed in tabular form and then in- terpolated in azimuth and orbit position to obtain values for each pulse and slice.

2) Spatial-Response Function and Surface Sampling: It is insightful to view the $X$ parameter as an integration of the instrument spatial-response function on the Earth's surface. The spatial-response function can be constructed from (21) by taking the energy contribution to the slice from each scattering patch and then normalizing by the area of the patch. Denoting this function as $S$, we have

$$
\begin{aligned}
S\left(\overrightarrow{l_{i}}\right)= & \frac{C^{2} g_{i}\left(t_{t r s}\right) g_{i}\left(t_{r e c}\right)}{T_{p} r_{i}^{4}} \\
& \cdot \sum_{k=k_{s}}^{k_{e}}\left[\frac{\sin ^{2}\left[\pi N_{p, i}\left(f_{b, i} T-\frac{k}{N}\right)\right]}{\sin ^{2}\left[\pi\left(f_{b, i} T-\frac{k}{N}\right)\right]}\right]
\end{aligned}
$$

where $\overrightarrow{l_{i}}$ is the surface position (in latitude and longitude, for instance) of the $i$ th patch.

In Fig. 10, the spatial-response function is displayed for two cases. In Fig. 10(a), the response for the beam-limited case (no range filtering) is shown. In Fig. 10(b), the response for an example range slice of width $7 \mathrm{~km}\left(B_{s}=8.3 \mathrm{kHz}\right.$ for $\mu=250$ $\mathrm{kHz} / \mathrm{ms}$ ) is shown. Here, it is clear that the measured cross-section will be an average of surface $\sigma^{\circ}$ weighted by the spatial

$$
X=\frac{C^{2}}{T_{p}} \sum_{i \in \mathcal{F}}\left\{\left(\frac{\delta A_{i} g_{i}\left(t_{t r s}\right) g_{i}\left(t_{r e c}\right)}{r_{i}^{4}}\right) \sum_{k=k_{s}}^{k_{e}} \cdot\left[\frac{\sin ^{2}\left[\pi N_{p, i}\left(f_{b, i} T-\frac{k}{N}\right)\right]}{\sin ^{2}\left[\pi\left(f_{b, i} T-\frac{k}{N}\right)\right]}\right]\right\} .
$$




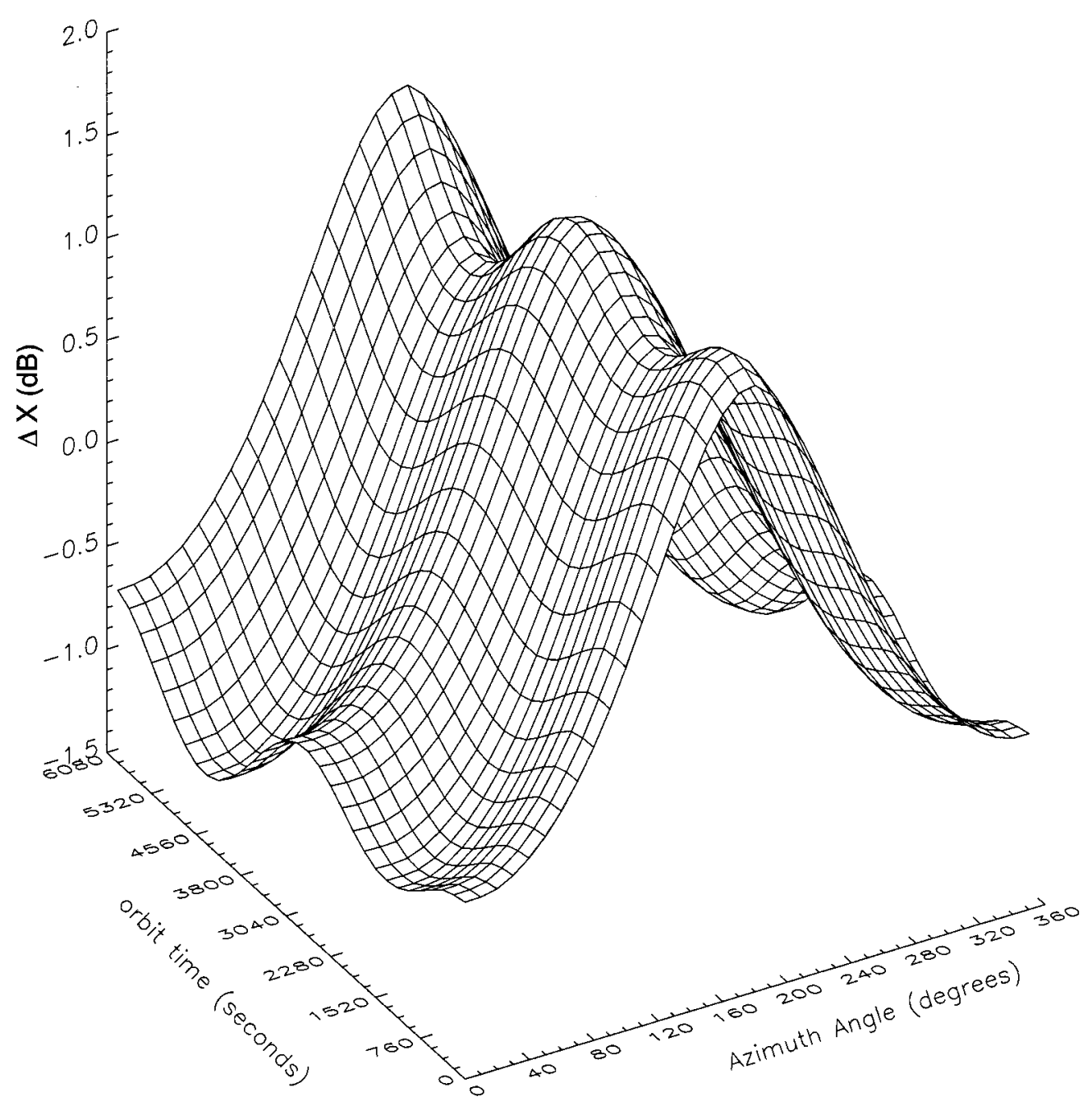

Fig. 9. $X$-parameter for example slice versus orbit time (one complete orbit $=6080 \mathrm{~s}$ ) and antenna-scan azimuth angle. Here, $X$ has been normalized by its mean value.

response function. In Fig. 10(b), note the sharp dropoff of the response function in the range direction. Such a sharp edge in the spatial response preserves high-frequency information in the spatial-frequency domain, and thus, it is a highly desirable property for enhanced-resolution, image reconstruction purposes. Note also the tilt in the orientation of the slice due to Doppler shift across the footprint. In Fig. 11, the surface sampling achieved by the center eight slices for several consecutive pulses is shown. The eight center slices correspond approximately to the extent of the antenna main beam and thus, they are the slices expected to yield measurements of sufficient accuracy. Here, the $\sigma^{\circ}$-cell outlines are delineated by plotting the approximate $3-\mathrm{dB}$ contour of the slice response. As is evident, the measurements form a very dense sampling of the surface with many overlapping cells. This is another property favorable for ERI [3], as well as higher resolution wind retrieval.

3) Errors in $X$ : Regardless of the care taken in deriving an expression for $X$, the calibration accuracy can only be as good as our knowledge of the various instrumental and geometric parameters comprising $X$. The parameter $C$ in (5) embodies all radiometric components of $X$. Although there is potential for error in determining the value of $C$, this error is likely to be a constant bias for all measurements and should not contribute significantly to relative calibration error. This is because the determination of transmit power and receiver gain through periodic "loop-back" calibration measurements relies on a very stable, thermally controlled waveguide coupler. Furthermore, the antenna gain and system losses are likewise expected to be quite constant because of reliance on equally stable passive RF components. What can change on-orbit, however, is the measurement geometry, primarily through variations in the spacecraft attitude. In fact, attitude-knowledge errors were observed to constitute the dominant source of relative calibration error for both the SEASAT-A and NSCAT scatterometers [7], [19].

As the attitude changes, the antenna pattern shifts with respect to the lines of constant baseband frequency on the Earth, which form the slice edges. If the attitude change is unknown, an error in $X$, and consequently an error in $\sigma^{\circ}$, will result. The magnitude of this error can be evaluated by taking the ratio of $X$ calculated at the true attitude to the estimated attitude. In gen- 


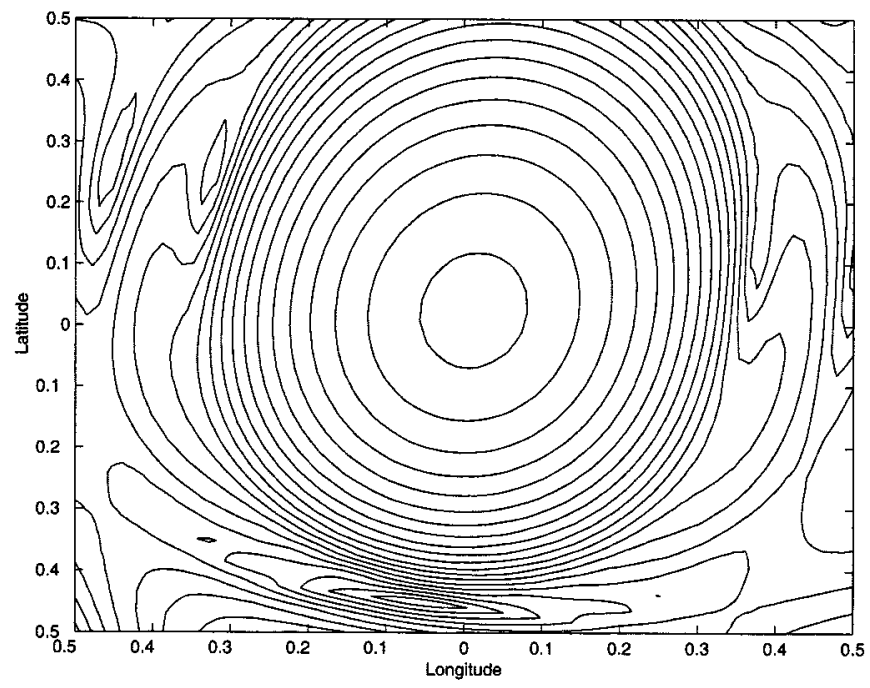

(a)

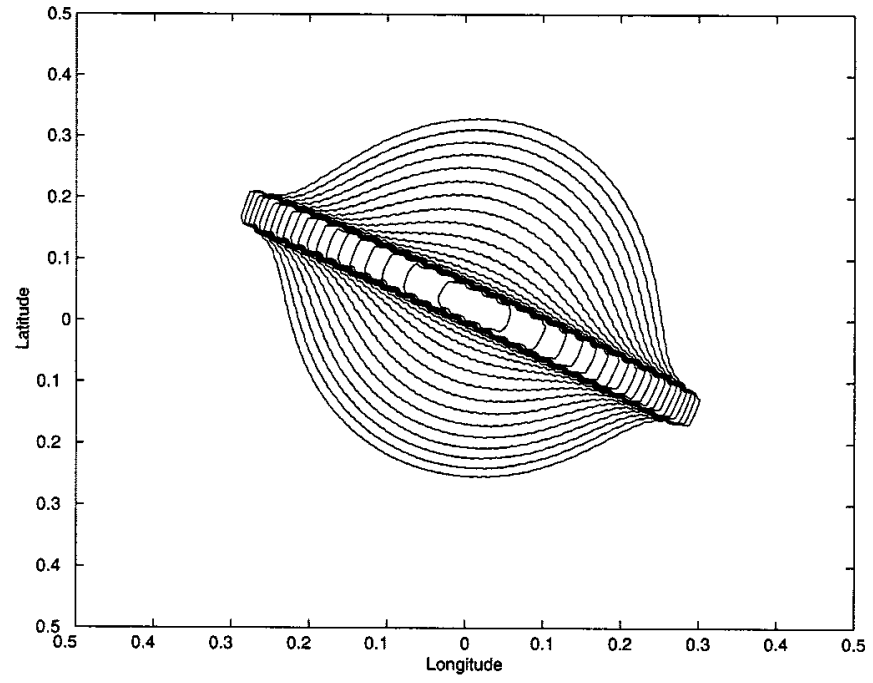

(b)

Fig. 10. (a) Example inner-beam, $\sigma^{\circ}$-cell spatial response for beam-limited (no range filtering) case. (b) Example inner-beam, $\sigma^{\circ}$-cell spatial response for center-range slice. Contour spacing at $1-\mathrm{dB}$ intervals.

eral, we are much more sensitive to attitude changes that effect the elevation angle of the antenna pointing (spacecraft pitch and roll) as opposed to changes that effect the azimuth angle (spacecraft yaw). In Fig. 12, the error in $\sigma^{\circ}$ is plotted versus the error in elevation angle for different slices. The slices are numbered according to their position in the antenna beam. Slice 1 is an "inner" slice near the peak of the antenna pattern as projected on the surface and slice 5 is an "outer" slice further down on the main beam. It is evident that slices near the peak (where the antenna pattern varies slowly) are relatively insensitive to changes in pointing, whereas the outer slices where the pattern is changing rapidly are quite sensitive to pointing errors. This, then, becomes a key design consideration for improved resolution measurements that did not exist for the low-resolution case. To achieve the desired calibration accuracy for all slices requires spacecraft pointing knowledge on the order of $0.02^{\circ}$. Less ambitious designs for the spacecraft attitude determination system, however, would imply that calibration goals may still be met for the innermost slices.

\section{B. Backscatter Measurement Variance}

As mentioned in Section II, measurements of the detected energy $P_{s}^{q}$ are "noisy" due to radar fading and the presence of system thermal noise. Unlike calibration error, which is essentially deterministic and can be improved by better knowledge of instrument parameter values and pointing, the random variations in $P_{s}^{q}$ place a fundamental limit on the instrument precision. For the selected range-filtering implementation, however, we may optimize measurement precision by careful selection of the transmit chirp rate and slice bandwidth. This section presents the various equations necessary to perform this optimization and discusses results for the SeaWinds design.

1) $K_{p}$ Parameter: Previous studies have addressed in detail the issue of measurement variance for fan-beam systems with Doppler filtering [2] and pencil-beam systems with transmit modulation and square-law detection [11]. Here, the analysis specific to a pencil-beam scatterometer with linear chirp modulation and digital range filtering is outlined.

In scatterometry, it has become customary to define the measurement error in terms of the so-called $K_{p}$ parameter

$$
K_{p}=\frac{\sqrt{\operatorname{Var}\left[\sigma^{\circ}\right]}}{\mathcal{E}\left[\sigma^{\circ}\right]}=\frac{\sqrt{\operatorname{Var}\left[P_{s}\right]}}{\mathcal{E}\left[P_{s}\right]}
$$

where the slice index " $n$ " has been dropped for notational simplicity. $K_{p}$ is then the normalized standard deviation of the measurement error or percentage error. A goal of scatterometer design is the minimization of $K_{p}$. From (1), we can write the variance of $P_{s}^{q}$ as

$$
\begin{aligned}
\operatorname{Var}\left[P_{s}\right]= & \operatorname{Var}\left[C_{s n}\right]+\operatorname{Var}\left[P_{n}\right] \\
& +2\left(\mathcal{E}\left[C_{s n}\right] \mathcal{E}\left[P_{n}\right]-\mathcal{E}\left[C_{s n} P_{n}\right]\right) .
\end{aligned}
$$

As is shown in [11], when $B_{n} \gg B_{s}$, the second and third terms of (24) are much smaller than $\operatorname{Var}\left[C_{s n}\right]$. For SeaWinds, because $B_{n}=1 \mathrm{MHz}$ and $B_{s}=8.3 \mathrm{kHz}$, this condition applies and allows us to assume

$$
K_{p}=\frac{\sqrt{\operatorname{Var}\left[C_{s n}\right]}}{\mathcal{E}\left[P_{s}\right]} .
$$

Because the derivation of $\operatorname{Var}\left[C_{s n}\right]$ is somewhat involved, only the major assumptions and results are discussed here. The derivation procedure is more similar to the development described in [11], which employs time domain techniques, rather than [2] which employs a frequency domain approach. This is to insure that the resultant expression is sufficiently general to handle the case where the echo return can not be accurately modeled as a stationary random process. Noting that $C_{s n}$ can be written as

$$
C_{s n}=C^{2} \sum_{k=k_{s}}^{k_{e}}\left|\sum_{n=0}^{N-1}(d[n]+\nu[n]) e^{-2 \pi j(k n / N)}\right|^{2}
$$




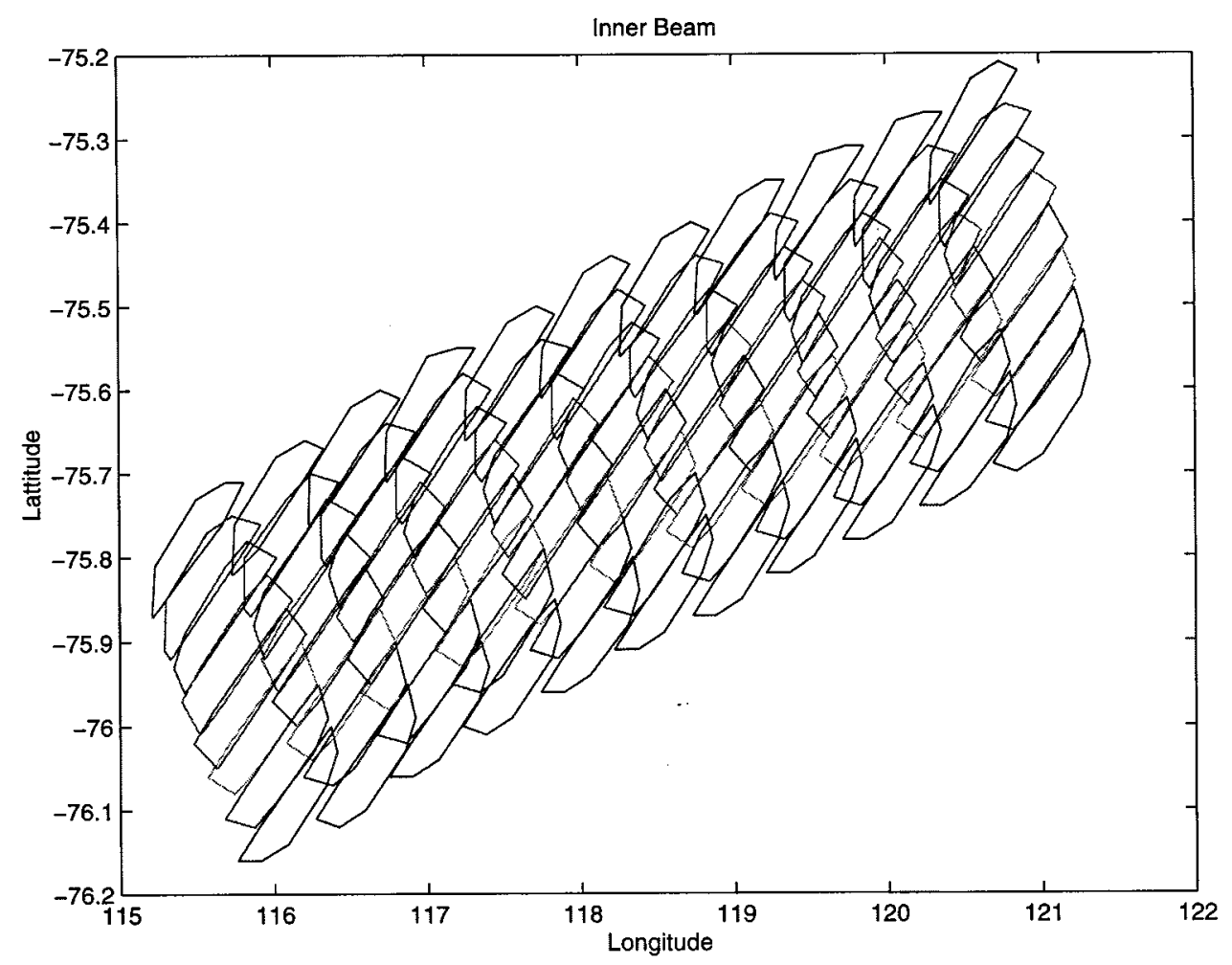

Fig. 11. Center eight-slice outlines for eleven consecutive transmit pulses for the inner antenna beam. Slice outlines are given by approximations of the 3-dB spatial-response contour.

where $d[n]$ is the signal sequence as described in (11), and $\nu[n]$ is the noise sequence, the necessary expectations of squared terms and associated cross products are then taken. Making the reasonable assumption that both signal and noise-voltage terms are Gaussian distributed, a familiar expansion for the fourthorder moment of Gaussian random variables is employed [11]. Further assuming that the return echo from a given scatterer has a rectangular envelope (the same assumption that was made to develop (21)) it can be shown that

$$
K_{p c}^{2}=A+\frac{B}{\mathrm{SNR}}+\frac{C}{\mathrm{SNR}^{2}} .
$$

Here, the SNR is defined as

$$
\mathrm{SNR}=\frac{X \sigma^{\circ}}{T_{g} B_{S} N_{0}}
$$

where $T_{g}$ is the range-gate length, and $N_{0}$ is the noise-floor power spectral density expressed in suitable units. The parame- ters $A, B$, and $C$ can be shown in (29), at the bottom of the page. Where $\beta(i, k)$ is as defined in (20), $n_{\nu}$ is the sample number in $d[n]$ corresponding to the opening of the range gate, $N_{\nu}$ is the duration (in number of samples) of the range-gate open time, $K$ is the total number of periodogram bins summed to form the slice, and $c_{i}$ is defined as

$$
c_{i}=\frac{C^{2} \delta A_{i} g_{i}\left(t_{t r s}\right) g_{i}\left(t_{r e c}\right)}{T_{p} r_{i}^{4}} .
$$

Equation (29) can be approximated by a form more suited to intuitive analysis by making the following assumptions:

1) echo return is nearly stationary, i.e., the pulse length $T_{p}$ $(1.5 \mathrm{~ms}$ for SeaWinds) is much greater than the time it takes the fill the entire antenna beam (about $0.3 \mathrm{~ms}$ for SeaWinds);

2) $B_{3 \mathrm{~dB}} \gg B_{s}$;

3) $T_{p} B_{s} \gg 1$.

$$
\begin{aligned}
& A=\frac{1}{X^{2}} \sum_{k} \sum_{h} \sum_{i} \sum_{l} c_{i}^{2} c_{l}^{2} \beta(i, k) \beta^{*}(i, h) \beta^{*}(l, k) \beta(l, h) \\
& B=\frac{2}{K N_{\nu} X} \sum_{k} \sum_{h} \sum_{i} c_{i}^{2} \frac{\exp \left(j \pi \frac{(k-h)}{N}\left(N_{\nu}+2 n_{\nu}\right)\right) \sin \left(\pi \frac{(k-h)}{N} N_{\nu}\right)}{\exp \left(j \pi \frac{(k-h)}{N}\right) \sin \left(\pi \frac{(k-h)}{N}\right)} \beta(i, k) \beta^{*}(i, h) \\
& C=\frac{1}{K^{2} N_{\nu}^{2}} \sum_{k} \sum_{h} \sum_{n=0}^{N_{\nu}-1} \sum_{m=0}^{N_{\nu}-1} \exp \left(j \frac{2 \pi}{N}(k-h)(m-n)\right)
\end{aligned}
$$




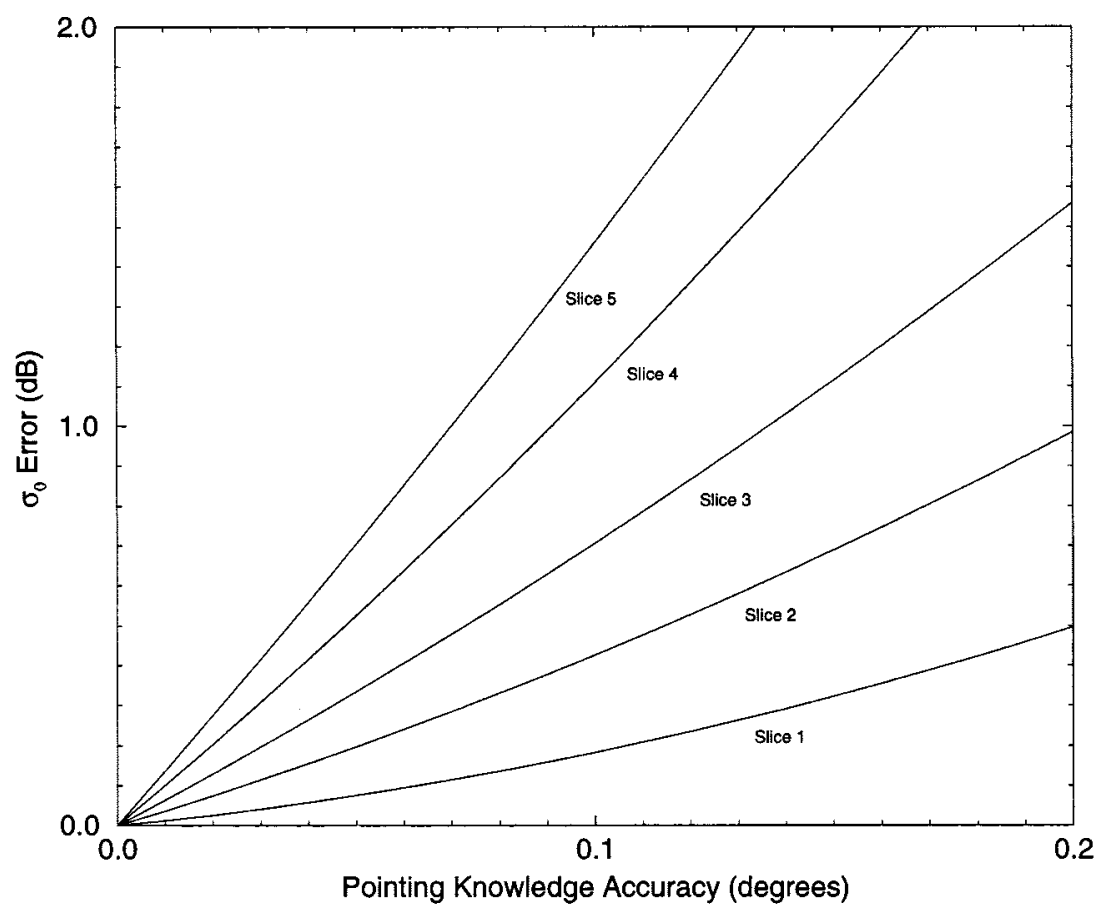

Fig. 12. $\sigma^{\circ}$ calibration (or, equivalently, $X$ error) as a function of antenna-pointing elevation-angle knowledge accuracy. Slice 1 is the innermost slice (closest to antenna beam center), and Slice 5 is the outermost slice (on edge of the antenna pattern).

Under these conditions, which apply for SeaWinds, we can approximate $A, B$, and $C$ as

$$
\begin{aligned}
A & =\frac{1}{B_{s} T_{p}} \\
B & =\frac{2}{B_{s} T_{g}} \\
C & =\frac{1}{B_{s} T_{g}} .
\end{aligned}
$$

Taking (31) together with (27) and (28), we obtain insight into the design considerations necessary to minimize $K_{p}$. The " $A$ " term is the contribution to the variance due to radar signal fading alone, with $B_{s} T_{p}$ approximating the number of independent "looks" associated with a given measurement slice. Assuming that $T_{p}$ is fixed due to timing and sampling constraints, we can only reduce $A$ by increasing the bandwidth of the slice measurement, $B_{s}$. The slice bandwidth is, in turn, related to the narrow (range) dimension of the slice spatial response on the surface and the transmit chirp rate. For the SeaWinds orbit altitude of $800 \mathrm{~km}$

$$
B_{s} \approx W \sqrt{2 \times 10^{-5} \mu^{2}+0.14}
$$

where $B_{s}$ is the slice bandwidth in $\mathrm{kHz}, \mu$ is the transmit-pulse chirp rate in $\mathrm{kHz} / \mathrm{ms}$, and $W$ is the mean-range dimension of the slice in $\mathrm{km}$. Thus, for a given slice dimension, we can increase the bandwidth by increasing the chirp rate. As discussed in [17], we desire to make $B_{s}$ as large as possible for a scanning scatterometer to compensate for the relatively short integration times ( $T_{p}$ and $T_{g}$ ), as opposed to the longer dwell times available with nonscanning, fan-beam systems.

A tradeoff exists, however, because as $B_{s}$ is increased, SNR decreases [see (28)], and the second and third terms of (27) get larger. In other words, we must balance the benefits of a larger measurement bandwidth with the effects of allowing more thermal noise to enter the measurement. This analysis is governed by a consideration of the backscatter strength from ocean winds, which generally have lower SNR than land targets. A similar tradeoff analysis was performed for the previous beam-limited SeaWinds design (outlined in [17]). Proceeding along similar lines, a chirp rate of $\mu=250 \mathrm{kHz} / \mathrm{ms}$ was selected for the new design with range filtering. This value was found to strike a balance in $K_{p}$ performance for high windspeeds (which have high inherent SNR $(>6 \mathrm{~dB})$ and hence, benefit from larger measurement bandwidths) and low windspeeds (which have low SNR $(<0 \mathrm{~dB})$, where the variance may be made worse by increasing the measurement bandwidth).

\section{Enhanced-Resolution Imaging Performance}

As one example of how the addition of range-filtering capability expands the utility of SeaWinds, in this section, we examine the resulting improved "land ice" imaging performance. As previously noted, $\mathrm{Ku}$-band scatterometer $\sigma^{\circ}$ measurements have proved to be very useful in land and ice studies (hence, the desire to maintain a long time series of such measurements). While the original design of SeaWinds would have provided usable measurements, the modified design will provide $\sigma^{\circ}$ measurements with significantly improved resolution. This is expected to extend the utility of the SeaWinds measurements in 


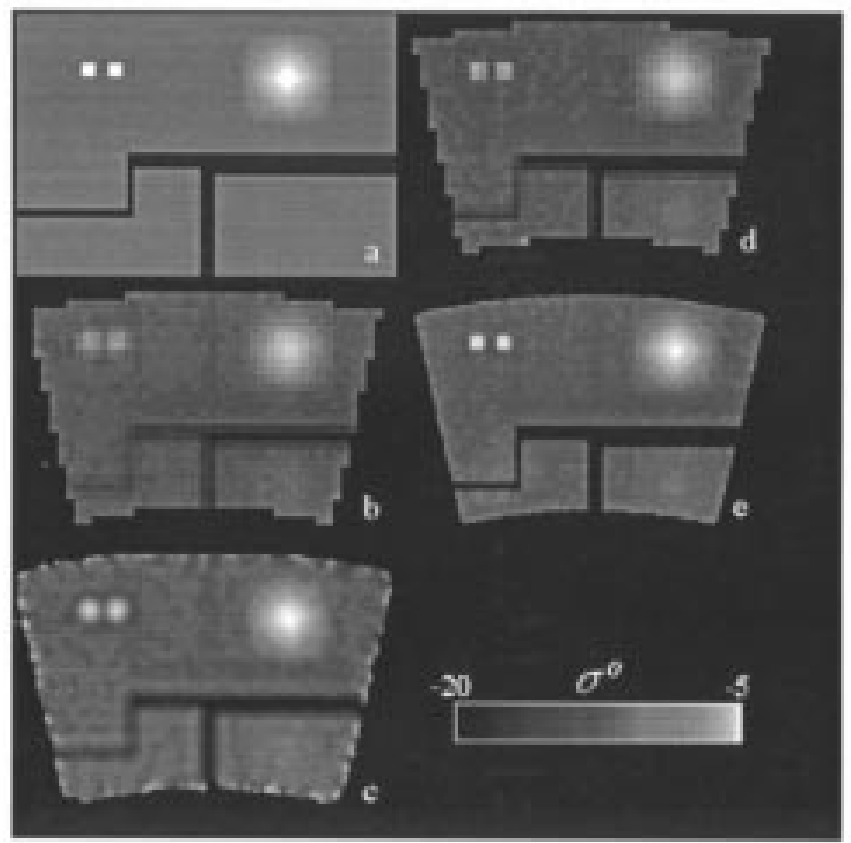

Fig. 13. Simulated images from QuikSCAT/SeaWinds. (a) Simulated truth image, (b) gridded image (25-km grid) using beam-limited footprint (no slicing), (c) SIR-enhanced resolution image using beam-limited footprint, (d) gridded image using the ten inner slices (25-km grid), and (e) SIRF-enhanced resolution image using slices. Pixel resolution is $4.5 \mathrm{~km}$. The area is a small, synthetic region in Wilkes Land, Antarctica (hence the odd shape, which is a box in latitudinal/longitudinal space but is mapped using a Lambert projection to a flat map).

land ice science studies, as well as for the primary wind observation mission. Here, the land ice imaging resolution of the original and modified SeaWinds designs are compared using conventional gridding and a particular ERI technique known as scatterometer image reconstruction with filtering (SIRF) [9].

To make the performance comparison, both beam-limited and range-sliced $\sigma^{\circ}$ cells are used. Simulated backscatter measurements are generated with the aid of a synthetic image of the surface $\sigma^{\circ}$ [see Fig. 13(a)], which is similar to that used in [9]. For each beam-limited footprint or range slice, the effective $\sigma^{\circ}$ measurement is computed as the weighted average of the pixels of the synthetic image, where the weighting is the spatial response function described earlier. Then, $K_{p}$ is computed, and Monte Carlo noise is added to generate a simulated noisy- $\sigma^{\circ}$ measurement. For this analysis, calibration errors were neglected.

In order to simulate the Earth location and orientation of the measurements, the synthetic test image was located over Wilkes Land, Antarctica. The test region is approximately $1000 \mathrm{~km} \mathrm{x}$ $800 \mathrm{~km}$ and is centered at $74.5^{\circ} \mathrm{S}$ and $128.5^{\circ} \mathrm{W}$. Over a one-day period, at least part of the test site is observed during five passes of QuikSCAT. The imaging results for a number of cases are compared in Fig. 13. Using the simulated $\sigma^{\circ}$ measurements, images were computed using a (nonenhanced) gridding approach and the SIRF resolution enhancement technique for both beamlimited cells and slices. The nonenhanced grid images have a pixel resolution of approximately $25 \mathrm{~km}$, while the SIRF images have a pixel resolution of approximately $4.5 \mathrm{~km}$.
To generate the nonenhanced images, each $\sigma^{\circ}$ measurement is assigned to the grid element in which its center falls. The average $\sigma^{\circ}$ is then computed and assigned to the associated pixel. The SIRF images were generated with a modified form of the SIRF algorithm. While the original SIRF algorithm (described in [9]) is bivariate, estimating both the incidence-angle normalized $\sigma^{\circ}$ and the incidence-angle dependence of $\sigma^{\circ}$, the algorithm used here is modified to image only $\sigma^{\circ}$, similar to the radiometer version of the algorithm [12].

Subjectively, the addition of range-resolution capability is observed to significantly improve the effective 2 -D resolution of land ice images produced from the simulated SeaWinds measurements whether or not resolution enhancement is applied. Even though range filtering resolves the footprint in just one dimension, this is true because of the different orientations of the $\sigma^{\circ}$ cells contributing to each pixel. Using the SIRF algorithm further improves the image resolution over the gridding approach. Because the SeaWinds measurements densely overlap, reasonable images can be made from only one day of data in this polar region. However, the noise level in the images can be reduced if multiple days are combined and the surface is temporally stable.

\section{SUMMARY}

Flights of the SeaWinds instrument in 1999 and 2000 form the foundation of the NASA Ku-band scatterometer program into the next century. In this paper, the new design for SeaWinds resolution processing has been described. As demonstrated, the spatial resolution performance of SeaWinds has been significantly improved by the addition of a range filtering scheme. This will be particularly useful for land and ice images constructed using enhanced-resolution imaging algorithms. Range slicing of the antenna footprint, coupled with the application of ERI, is thus an economical way of extending the capabilities of small, scanning pencil-beam scatterometers such as SeaWinds.

As discussed, range filtering is generally preferred over Doppler filtering because of superior measurement variance performance and more favorable geometrical orientation of the $\sigma^{\circ}$ cells. Although range discrimination by deramp processing is a relatively straightforward approach, its implementation must be accompanied by a careful accounting of geometric calibration factors in order to meet backscatter measurement accuracy goals. This involves a formulation for the radar equation, which includes digital processing and antenna rotation effects, as well as a consideration of spacecraft attitude variations. Tradeoffs to obtain minimum-measurement variance by optimizing the chirp rate and detection bandwidth for the available SNR must also be conducted to obtain the best performance.

\section{APPENDIX \\ Calculating $P_{n}^{q}$}

As discussed in Section II, to determine $\sigma^{\circ}$ from each measurement slice, we must first subtract the thermal-noise energy component $P_{n}^{q}$. To determine $P_{n}^{q}$, we use both the noise-only processor output $C_{n o}$ and the sum of all the slice measurements $C_{s n}^{e}$, where $C_{s n}^{e}=\sum_{q=1}^{12} C_{s n}^{q}$. 
To insure that the thermal-noise estimate corresponds to the same surface scene observed during the slice measurement, the noise-only and signal + noise processing operations are gated simultaneously (see [17]). Thus, we have

$$
\begin{aligned}
& C_{n o}=g_{n}\left(E+B_{n} T_{g} N_{0}\right) \\
& C_{s n}^{e}=g_{e}\left(E+B_{e} T_{g} N_{0}\right)
\end{aligned}
$$

where $g_{n}$ and $g_{e}$ are the receiver gains through the noise-only and signal + noise processing paths, respectively, $E$ is the total echo energy captured during gate time $T_{g}, B_{n}$ and $B_{e}$ are the effective bandwidths of the two measurements (see Fig. 7), and $N_{0}$ is the thermal-noise power spectral density. Here, it is assumed that $B_{e}$ is sufficiently wide to pass all the deramped echo power spectrum.

Eliminating $E$ and solving for $g_{e} T_{g} N_{0}$, we obtain

$$
g_{e} T_{g} N_{0}=\left(\frac{1}{B_{e}}\right) \frac{\frac{g_{e}}{g_{n}} C_{n o}-C_{s n}^{e}}{\frac{B_{n}}{B_{e}}-1}
$$

which is the total thermal-noise contribution in the bandwidth $B_{e}$. Note that to get this result, we needed only the ratio of the gains in the two channels and not their numerical values. Because we know $B_{s} / B_{e}$, we can obtain

$$
P_{n}^{q}=B_{s}^{q} g_{e} T_{g} N_{0}
$$

\section{REFERENCES}

[1] E. P. W. Attema, "The active microwave instrument on-board the ERS-1 satellite," Proc. IEEE, vol. 79, pp. 791-799, June 1991.

[2] C.-Y. Chi, D. G. Long, and F. K. Li, "Radar backscatter measurement accuracies using digital Doppler processors in spaceborne scatterometers," IEEE Trans. Geosci. Remote Sensing, vol. GE-24, pp. 426-437, May 1986

[3] D. Early, "A study of the scatterometer image reconstruction algorithm and its applications to polar ice studies," Ph.D. dissertation, Brigham Young Univ., Provo, UT, 1998.

[4] M. H. Freilich, D. G. Long, and R. S. Dunbar, "NSCAT measurement of wind wakes and jets near South Georgia Island," Science, 1998, to be published.

[5] J. E. Graf, W.-Y. Tsai, and W. L. Jones, "Overview of the QuikSCAT mission - A quick deployment of a high resolution, wide swath scanning scatterometer for ocean wind measurement," in Southeastcon'98, Orlando, FL, Apr. 1998.

[6] W. L. Grantham, E. M. Bracalente, W. L. Jones, and J. W. Johnson, "The Seasat-A satellite scatterometer," IEEE J. Oceanic Eng., vol. OE-2, pp. 200-206, Apr. 1977.

[7] J. W. Johnson et al., "SEASAT-A satellite scatterometer instrument evaluation," IEEE J. Ocean. Eng., vol. OE-5, pp. 138-144, 1980.

[8] W. L. Jones, V. J. Cardone, W. J. Pierson, J. Zec, L. P. Rice, A. Cox, and W. B. Sylvester, "NSCAT high resolution surface winds measurements in typhoon Violet," J. Geophys. Res.-Oceans (Special NSCAT issue), to be published.

[9] D. G. Long, P. J. Hardin, and P. T. Whiting, "Resolution enhancement of spaceborne scatterometer data," IEEE Trans. Geosci. Remote Sensing, vol. 31, pp. 700-715, May 1993.
[10] D. G. Long and P. Hardin, "Vegetation studies of the Amazon Basin using enhanced resolution Seasat scatterometer data," IEEE Trans. Geosci. Remote Sensing, vol. 32, pp. 449-460, Mar. 1994.

[11] D. G. Long and M. W. Spencer, "Radar backscatter measurement accuracy for a spaceborne pencil-beam wind scatterometer with transmit modulation," IEEE Trans. Geosci. Remote Sensing, vol. 35, pp. 102-114, Jan. 1997.

[12] D. G. Long and D. L. Daum, "Spatial resolution enhancement of SSM/I data," IEEE Trans. Geosci. Remote Sensing, vol. 35, pp. 407-417, Mar. 1998.

[13] D. G. Long and M. R. Drinkwater, "Cryosphere studies using NSCAT data," IEEE Trans. Geosci. Remote Sensing, vol. 37, pp. 1671-1684, May, 1999.

[14] R. K. Moore, "Effect of pointing errors and range on performance of dual-pencil-beam scatterometers," IEEE Trans. Geosci. Remote Sensing, vol. GE-23, pp. 901-905, Nov. 1985.

[15] F. Naderi, M. H. Freilich, and D. G. Long, "Spaceborne radar measurement of wind velocity over the ocean-An overview of the NSCAT scatterometer system," Proc. IEEE, vol. 79, pp. 850-866, June 1991.

[16] W. D. Robinson, C. Kummerow, and W. S. Olson, "A technique for enhancing and matching the resolution of microwave measurements from the SSM/I instrument," IEEE Trans. Geosci. Remote Sensing, vol. 30, pp. 419-429, May 1992.

[17] M. W. Spencer, C. Wu, and D. G. Long, "Tradeoffs in the design of a spaceborne scanning pencil beam scatterometer: Application to SeaWinds," IEEE Trans. Geosci. Remote Sensing, vol. 35, pp. 115-126, Jan. 1997.

[18] J. N. Huddleston, W.-Y. Tsai, M. W. Spencer, and R. D. West, "Modeling and simulation for SeaWinds 1-B system design and performance evaluation," in Proc. SPIE: Sensors, Systems, and Next-Generation Satellites, London, U.K., Sept. 1997.

[19] W.-Y. Tsai, J. E. Graf, C. Winn, J. N. Huddleston, R. S. Dunbar, M. H. Freilich, F. J. Wentz, D. G. Long, and W. L. Jones, "Post-launch sensor verification and calibration of the NASA scatterometer," IEEE Trans. Geosci. Remote Sensing, vol. 37, pp. 1517-1542, May 1999, to be published.

[20] F. T. Ulaby, R. K. Moore, and A. K. Fung, Microwave Remote Sensing-Active and Passive. Reading, MA: Addison-Wesley Publishing Company, 1981, vol. 2

[21] V. R. Wismann and K. Boehnke, "Monitoring snow properties on Greenland with ERS scatterometer and SAR," in Proc. 3rd ERS Symp. ESA SP-414, vol. 2, May 1997, pp. 857-886.

[22] C. Wu, J. Graf, M. Freilich, D. G. Long, M. Spencer, W. Tsai, D. Lisman, and C. Winn, "The SeaWinds scatterometer instrument," in Proc. Int Geoscience and Remote Sensing Symp., Pasadena, CA, Aug. 8-12, 1994, pp. 1511-1515.

[23] A. R. Zieger, D. W. Hancock, G. S. Hayne, and C. L. Purdy, "NASA radar altimeter for the TOPEX/POSEIDON project," Proc. IEEE, vol. 79, pp. 810-825, June 1991.

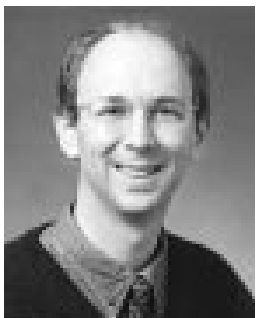

Michael W. Spencer received the B.S. degree in physics from the College of William and Mary, Williamsburg, VA, in 1985, and the M.S. degree in planetary science from the California Institute of Technology, Pasadena, in 1987. Through an employer-sponsored program, he later received the M.S.E.E. degree from the University of Southern California, Los Angeles, and is currently pursuing the Ph.D. degree in electrical and computer engineering at Brigham Young University, Provo, UT.

From 1987 to 1990 , he was with the Aerospace Corporation, Los Angeles, CA, where he was involved in the modeling and analysis of advanced meteorological and surveillance satellite systems. Since 1990, he has been employed at the Jet Propulsion Laboratory, Pasadena, CA, where he has worked on the conceptualization, system design, simulation, and calibration of radar remote sensing instruments. His general research interests also include statistical signal processing and data visualization.

Mr. Spencer is a member of Phi Beta Kappa. 


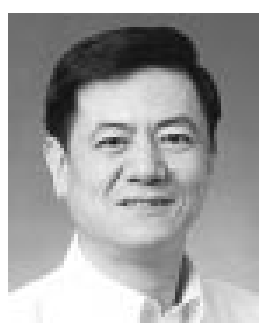

Chialin Wu was born in Nanjing, China, in 1947. He received the B.S. in physics from the National Taiwan University, Taipei, in 1969, and subsequently the M.S. and Ph.D. degrees in electrical engineering from Purdue University, West Lafayette, IN, in 1972 and 1975 , respectively.

While at Purdue University, he did research in imaging sonar and remote sensing. In the remote-sensing area, he was involved in the NASA ERTS (the early LANDSAT) satellite multispectral-pattern recognition and image processing tasks at the Laboratory for Application of Remote Sensing. In 1974, he joined the Jet Propulsion Laboratory, and engaged in digital signal processing and system engineering for the Seasat SAR. He developed the signal-processing algorithm and also the operational-processor system for producing digital SAR imagery from the 1978 Seasat mission. After the Seasat mission, he was the Co-Investigator of a JPL research task to develop spaceborne SAR interferometry. This research led to a patent and a NASA monetary award in 1987. He served on the Magellan Mission to Venus as the Deputy Manager for the Radar Office from 1989 to 1992, during the cruise and radar mapping period, and was responsible for radar system engineering and operation. Since 1993, he has supported the JPL spaceborne radar scatterometer project as the Experiment Engineer for SeaWinds and is responsible for the radar design requirements, end-to-end performance, and validation testing.

Dr. Wu received the NASA Exceptional Service Medal for the Seasat SAR processor development in 1978.

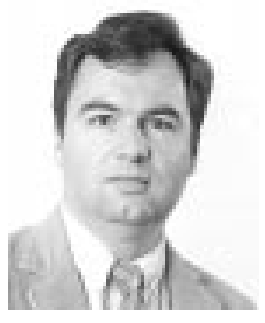

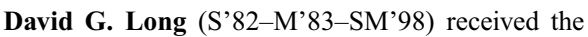
$\mathrm{Ph} . \mathrm{D}$. degree in electrical engineering from the University of Southern California, Los Angeles, in 1989.

From 1983 to 1990 , he was with NASA's Jet Propulsion Laboratory (JPL), Washington, DC, where he developed advanced radar remote sensing systems. While at the JPL, he was the Senior Project Engineer on the NASA Scatterometer (NSCAT) Project. NSCAT was flown aboard the Japanese Advanced Earth Observing System (ADEOS) from 1996 to 1997 . He is currently a Professor in the Electrical and Computer Engineering Department, Brigham Young University, Provo, UT, where he teaches upper division and graduate courses in communications, microwave remote sensing, radar, and signal processing. He is the principle investigator on several NASA-sponsored interdisciplinary research projects in remote sensing, including innovative radar systems, spaceborne scatterometry of the ocean and land, and modeling of atmospheric dynamics. He is a member of the NSCAT Science Working Team and has numerous publications in signal processing and radar scatterometry. His research interests include microwave remote sensing, radar theory, space-based sensing, estimation theory, computer graphics, signal processing, and mesoscale atmospheric dynamics.. 